\title{
Backward stochastic differential equations with unbounded generators
}

\author{
Bujar Gashi and Jiajie $\mathrm{Li}^{1}$ \\ Institute of Financial and Actuarial Mathematics (IFAM), \\ Department of Mathematical Sciences, The University of Liverpool, Liverpool, L69 7ZL, UK \\ Emails: Bujar.Gashi@liverpool.ac.uk; Jiajie.Li@me.com
}

\begin{abstract}
In this paper we consider two classes of backward stochastic differential equations. Firstly, under a Lipschitz-type condition on the generator of the equation, which can also be unbounded, we give sufficient conditions for the existence of a unique solution pair. The method of proof is that of Picard iterations and the resulting conditions are new. We also prove a comparison theorem. Secondly, under the linear growth and continuity assumptions on the possibly unbounded generator, we prove the existence of the solution pair. This class of equations is more general than the existing ones.
\end{abstract}

Keywords: BSDEs, Unbounded generator, Existence, Uniqueness, Comparison theorem.

\section{Introduction}

Let $\left(\Omega, \mathscr{F},\left(\mathscr{F}_{t}, t \geq 0\right), \mathbb{P}\right)$ be a given complete filtered probability space on which a $k$-dimensional standard Brownian motion $(W(t), t \geq 0)$ is defined. We assume that $\mathscr{F}_{t}$ is the augmentation of $\sigma\{W(s): 0 \leq s \leq t\}$ by all the P-null sets of $\mathscr{F}$. Consider the backward stochastic differential equation (BSDE):

$$
y(t)=\xi+\int_{t}^{T} f(s, y(s), z(s)) d s-\int_{t}^{T} z(s) d W(s), \quad t \in[0, T],
$$

where $\xi$ is a given $\mathscr{F}_{T}$-measurable $\mathbb{R}^{d}$-valued random variable, and the generator $f: \Omega \times(0, T) \times \mathbb{R}^{d} \times \mathbb{R}^{d \times k} \rightarrow \mathbb{R}^{d}$ is a progressively measurable function.

\footnotetext{
${ }^{1}$ Corresponding author.
} 
Linear equations of the type (10) were introduced by Bismut [5] in the context of stochastic linear quadratic control. The problem of existence and uniqueness of solution to the nonlinear equations (1) was solved by Pardaoux and Peng [34] under the global Lipschitz condition on $f$, i.e. under the assumption that there exists a real constant $c>0$ such that

$$
\left|f\left(t, y_{1}, z_{1}\right)-f\left(t, y_{2}, z_{2}\right)\right| \leq c\left(\left|y_{1}-y_{2}\right|+\left|z_{1}-z_{2}\right|\right)
$$

for all $y_{1}, y_{2} \in \mathbb{R}^{d}, z_{1}, z_{2} \in \mathbb{R}^{d \times k},(t, \omega)$ a.e.. Since then, the BSDEs have been studied extensively, and have found wide applicability in areas such as mathematical finance, stochastic control, and stochastic controllability; see, for example, [10], [17], [29], [31], [42], [37], [20], [21], [22] [41], and the references therein. One direction of research has been to weaken the assumption of global Lipschitz condition (2) by assuming only local Lipschitz condition (see [1]), or non-Lipschitz condition of a particular form (see [30], [39]). In [16] and [12] the authors permit for the generator function $f$ to be unbounded (they also consider a more general driving process than the Brownian motion). They assume the following global Lipschitz-type condition: there exist non-negative processes $c_{1}(\cdot)$ and $c_{2}(\cdot)$ such that

$$
\left|f\left(t, y_{1}, z_{1}\right)-f\left(t, y_{2}, z_{2}\right)\right| \leq c_{1}(t)\left|y_{1}-y_{2}\right|+c_{2}(t)\left|z_{1}-z_{2}\right|,
$$

for all $y_{1}, y_{2} \in \mathbb{R}^{d}, z_{1}, z_{2} \in \mathbb{R}^{d \times k},(t, \omega)$ a.e.. Clearly, this condition has great similarity with (2). However, different from (2), here the processes $c_{1}(\cdot)$ and $c_{2}(\cdot)$ are not assumed to be bounded. The linear BSDEs with possibly unbounded coefficients are considered in [43], using a very different approach as compared to [16], [12]. The interest in these equations is not only theoretical, but is also motivated by applications in mathematical finance. Indeed, some very important interest rate models are given by stochastic differential equations (see, for example, [44], [11], [4]). The problem of market completeness (and thus of pricing and hedging of derivatives) in such models gives rise to BSDEs with possibly unbounded coefficients (see [43] for details).

The first contribution of the present paper, as contained in the section 2, is to consider the problem of existence and uniqueness of a solution pair $(y(\cdot), z(\cdot))$ for (1) under condition (3). We do so under certain new conditions on the coefficients $c_{1}(\cdot), c_{2}(\cdot)$, which are similar to those of [16], but in general are not comparable. Moreover, our method of proof is different, since it is a modification of the Picard iteration procedure of [34] rather than being based on a fixed point theorem as in [16]. We also give a comparison theorem for 
this class of equations. This generalises the classical result of Peng [35], [36], to the case of BSDEs with possibly unbounded coefficients.

Another important weakening of the assumptions on the generator, as compared to [34], was given in [27] (see also [23]). There it assumed that the generator is continuous with respect to $y$ and $z$, and it satisfies the linear growth condition

$$
|f(t, y, z)| \leq c(1+|y|+|z|)
$$

for all $y \in \mathbb{R}, z \in \mathbb{R}^{k},(t, \omega)$ a.e.. Under such conditions, it was shown that equation (11) admits a solution pair. In more recent papers [39], [40], the linear growth condition (4) has been generalised to

$$
|f(t, y, z)| \leq c[q(t)+|y|+|z|],
$$

for all $y \in \mathbb{R}, z \in \mathbb{R}^{k},(t, \omega)$ a.e.. Here, different from (4), the process $q(\cdot)$ is not assumed to be bounded.

The generator of BSDEs with a quadratic growth on the control variable $z$ satisfies the condition:

$$
|f(t, y, z)| \leq k_{0}+k_{1}|y|+k_{2}|z|^{2} .
$$

An existence result for these equations was first given in [19] where $k_{0}$ and $k_{1}$ are constant, $k_{2}$ is a given function of $y$, and the terminal value $\xi$ is assumed bounded. In [6], [7], the assumption of a bounded $\xi$ was replaced with an integrability condition on the exponential of $\xi, k_{0}$ was permitted to be an unbounded process, whereas $k_{1}$ and $k_{2}$ were assumed constant (see further, for example, [2], [8], [9], [13], [14], [45], where a nonlinear growth in $y$ is also permitted in some cases). In [3], [18], [32], [33], the coefficient $k_{1}$ could also be an unbounded process. Under further assumptions on $f$, the uniqueness of the solution pair has been proved (see, for example, [9], [13], [14]). We are not aware of an existence result for these types of BSDEs with coefficient $k_{2}$ being an unbounded process.

The second contribution of the present paper, which is contained in section 3, is to consider a generator which is continuous in $y$ and $z$, but with a weaker linear growth condition than (5). We assume that

$$
|f(t, y, z)| \leq c_{0}(t)+c_{1}(t)|y|+c_{2}(t)|z|,
$$

for all $y \in \mathbb{R}, z \in \mathbb{R}^{k},(t, \omega)$ a.e.. Here the processes $c_{0}(\cdot), c_{1}(\cdot), c_{2}(\cdot)$, are not assumed to be bounded. By using the results of section 2, and appropriately 
modifying the approach of [2], [27], we prove the existence of a solution pair for (11). Note that due to the unbounded nature of the process $c_{2}(t)$, conditions (6) and (7) are in general not comparable if $k_{2}$ is a constant.

We conclude this introductory section with some notations:

- $|\cdot|$ is the Euclidian norm.

- $c_{0}(\cdot), c_{1}(\cdot), c_{2}(\cdot)$ are given $\mathbb{R}$-valued progressively measurable processes.

- $\gamma(\cdot), \overline{\gamma(\cdot)}$ and $\widetilde{\gamma(\cdot)}$ are given $\mathbb{R}$-valued positive progressively measurable processes.

- $1<\beta_{1}, \widetilde{\beta}_{1} \in \mathbb{R}, 1<\beta_{2}, \widetilde{\beta_{2}} \in \mathbb{R}$, are given constants.

- $4<\bar{\beta}_{1} \in \mathbb{R}, 1<90{\overline{\beta_{1}}}^{2} /\left({\overline{\beta_{1}}}^{2}-16\right)<\overline{\beta_{2}} \in \mathbb{R}$, are given constants.

- $\alpha_{1}(t) \equiv \gamma(t)+\beta_{1} c_{1}^{2}(t)+\beta_{2} c_{2}^{2}(t), \alpha_{2}(t) \equiv \overline{\gamma(t)}+\overline{\beta_{1}} c_{1}(t)+\overline{\beta_{2}} c_{2}^{2}(t)$, and $\widetilde{\alpha}(t) \equiv \widetilde{\gamma(t)}+\widetilde{\beta}_{1} c_{1}^{2}(t)+\widetilde{\beta}_{2} c_{2}^{2}(t)$ are assumed positive.

- $p_{1}(t) \equiv \exp \left[\int_{0}^{t} \alpha_{1}(s) d s\right], p_{2}(t) \equiv \exp \left[\int_{0}^{t} \alpha_{2}(s) d s\right]$ and $\widetilde{p}(t) \equiv \exp \left[\int_{0}^{t} \widetilde{\alpha}(s) d s\right]$.

- $L_{\mathscr{F}}\left(0, T ; \mathbb{R}^{d}\right)$ is the space of $\mathscr{F}{ }$-progressively measurable $\mathbb{R}^{d}$-valued processes $\varphi(\cdot)$ such that $\mathbb{E} \int_{0}^{T}|\varphi(t)| d t<\infty$.

- $M^{2}\left(\Omega, \mathscr{F}_{T}, \mathbb{P} ; \mathbb{R}^{d}\right)$ is the space of all $\mathscr{F}_{T}$-measurable $\mathbb{R}^{d}$-valued random variables $\zeta$ such that $\mathbb{E}\left[|\zeta|^{2}\right]<\infty$.

- $M^{2}\left(0, T ; \mathbb{R}^{d}\right)$ is the space of $\mathscr{F}{ }$-progressively measurable $\mathbb{R}^{d}$-valued processes $\varphi(\cdot)$ such that $\mathbb{E} \int_{0}^{T}|\varphi(t)|^{2} d t<\infty$.

- $\widehat{M}_{i}^{2}\left(\Omega, \mathscr{F}_{T}, \mathbb{P} ; \mathbb{R}^{d}\right)\left(\operatorname{resp} . \quad \widetilde{M^{2}}\left(\Omega, \mathscr{F}_{T}, \mathbb{P} ; \mathbb{R}^{d}\right)\right)$ is the space of all $\mathscr{F}_{T^{-}}$ measurable $\mathbb{R}^{d}$-valued random variables $\xi$ such that $\mathbb{E}\left[p_{i}(T)|\xi|^{2}\right]<\infty$ (resp. $\left.\mathbb{E}\left[\widetilde{p}(T)|\xi|^{2}\right]<\infty\right), i=1,2$.

- $\widehat{M}_{i}^{2}\left(0, T ; \mathbb{R}^{d}\right)\left(\right.$ resp. $\left.\widetilde{M^{2}}\left(0, T ; \mathbb{R}^{d}\right)\right)$ is the space of $\mathscr{F}_{t}$-progressively measurable $\mathbb{R}^{d}$-valued processes $\varphi(\cdot)$ such that $\mathbb{E} \int_{0}^{T} p_{i}(t)|\varphi(t)|^{2} d t<\infty$ (reps. $\left.\widetilde{\|\varphi\|} \equiv \mathbb{E} \int_{0}^{T} \widetilde{p}(t)|\varphi(t)|^{2} d t<\infty\right), i=1,2$. 
- $\widehat{H}_{i}^{2}\left(0, T ; \mathbb{R}^{d}\right)$ (resp. $\left.\widetilde{H}^{2}\left(0, T ; \mathbb{R}^{d}\right)\right)$ is the space of càdlàg $\mathscr{F}_{t}$-adapted $\mathbb{R}^{d}$-valued processes $\varphi(\cdot)$ such that $\mathbb{E}\left[\sup _{t \in[0, T]} p_{i}(t)|\varphi(t)|^{2}\right]<\infty$ (resp.

$\left.\mathbb{E}\left[\sup _{t \in[0, T]} \widetilde{p}(t)|\varphi(t)|^{2}\right]<\infty\right), i=1,2$.

\section{Unbounded Lipschitz-type generator}

In this section, we give sufficient conditions for the existence and uniqueness of a solution pair for (1). We say that the progressively measurable function $f$ and the random variable $\xi$, or the pair $(f, \xi)$, satisfies conditions A1 (resp. conditions A2) if:

(i) $\xi \in \widehat{M}_{1}^{2}\left(\Omega, \mathscr{F}_{T}, \mathbb{P} ; \mathbb{R}^{d}\right)\left(\operatorname{resp} . \xi \in \widehat{M}_{2}^{2}\left(\Omega, \mathscr{F}_{T}, \mathbb{P} ; \mathbb{R}^{d}\right)\right)$;

(ii) $\left|f\left(t, y_{1}, z_{1}\right)-f\left(t, y_{2}, z_{2}\right)\right| \leq c_{1}(t)\left|y_{1}-y_{2}\right|+c_{2}(t)\left|z_{1}-z_{2}\right|$, for all $y_{1}, y_{2} \in \mathbb{R}^{d}$, $z_{1}, z_{2} \in \mathbb{R}^{d \times k},(t, \omega)$ a.e.;

(iii) $\left[f(\cdot, 0,0) \alpha_{1}(\cdot)^{-\frac{1}{2}}\right] \in \widehat{M}_{1}^{2}\left(0, T ; \mathbb{R}^{d}\right)\left(\operatorname{resp} .\left[f(\cdot, 0,0) \alpha_{2}(\cdot)^{-\frac{1}{2}}\right] \in \widehat{M}_{2}^{2}\left(0, T ; \mathbb{R}^{d}\right)\right)$.

The sufficient conditions for the solvability of (11), as given in [16], are similar to our conditions A2. Indeed, if we choose $\bar{\gamma}(t)=0, \bar{\beta}_{1}=\bar{\beta}_{2} \equiv \beta$, where $\beta$ is large enough, then conditions A2 are those of [16]. Clearly, due to the process $\bar{\gamma}(t)$ our conditions A2 are more general then those of [16]. The importance of this process is that assumption (iii) above can be suitable weakened by choosing large values for this process, which is not an option in [16]. Moreover, even if we take $\bar{\gamma}(t)=0$, our assumption (i) is weaker than that of [16]. Indeed, the parameter $\beta$ of [16] should be bigger than 446.05 (in [16] it is only claimed that this coefficient should be large enough 2 ). This is clearly not the case in conditions $\mathrm{A} 2$ where the coefficient $\bar{\beta}_{1}$ is only required to be greater than 4 .

The conditions A1 are new. In general, these are not comparable with conditions A2. However, in certain special cases we can compare them. For

\footnotetext{
${ }^{2}$ For readers' convenience only, we have included an appendix showing that a straightforward calculation gives this numerical lower bound
} 
example, if $c_{1}(t)=0,1<\beta_{2}<\bar{\beta}_{2}, \gamma(t)=\bar{\gamma}(t)$, then $\widehat{M}_{1}^{2}\left(\Omega, \mathscr{F}_{T}, \mathbb{P}_{\mathbb{R}^{d}}\right) \subset$ $\widehat{M}_{2}^{2}\left(\Omega, \mathscr{F}_{T}, \mathbb{P} ; \mathbb{R}^{d}\right)$, and thus the above assumption (i) on the random variable $\xi$ is weaker in the case of conditions A1. Similarly, if $c_{2}(t)=0, \gamma(t)=\bar{\gamma}(t)$, $\bar{\beta}_{1}=2 \beta_{1}$, then the above assumption (i) on the random variable $\xi$ is weaker in the case of conditions A2.

\subsection{Solvability}

In this section we give sufficient conditions for the existence and uniqueness of a solution pair for (11). Our method of proof is different from [16] being based on Picard iterations, and similarly to [34], we begin with a simpler form of (11) and progress towards the general case. The proofs of the results under conditions A1 and A2 are different and are thus given separately in most cases, but there are also similarities between them.

Lemma 2.1. Let $\phi(\cdot) \in \widehat{H}_{1}^{2}\left(0, T ; \mathbb{R}^{d}\right), \psi(\cdot) \in \widehat{M}_{1}^{2}\left(0, T ; \mathbb{R}^{d \times k}\right)$ be given, and assume that $\sqrt{\alpha_{1}(\cdot)} \phi(\cdot) \in \widehat{M}_{1}^{2}\left(0, T ; \mathbb{R}^{d}\right)$. If the pair $(f, \xi)$ satisfies the conditions A1, then:

(i) there exists a unique solution pair $(y(\cdot), z(\cdot)) \in \widehat{H}_{1}^{2}\left(0, T ; \mathbb{R}^{d}\right) \times \widehat{M}_{1}^{2}\left(0, T ; \mathbb{R}^{d \times k}\right)$ of equation

$$
y(t)=\xi+\int_{t}^{T} f(s, \phi(s), \psi(s)) d s-\int_{t}^{T} z(s) d W(s), \quad t \in[0, T],
$$

and $\sqrt{\alpha_{1}(\cdot)} y(\cdot) \in \widehat{M}_{1}^{2}\left(0, T ; \mathbb{R}^{d}\right)$

(ii) if $y^{+}(t) \equiv \mathbb{1}_{[y(t)>0]} y(t)$, the processes

$$
\int_{t}^{T} p_{1}(s) y(s) z(s) d W(s) \quad \text { and } \quad \int_{t}^{T} p_{1}(s) y^{+}(s) z(s) d W(s),
$$

are martingales.

Proof. (i) By making use of the Cauchy-Schwartz inequality, we first show 
that $\int_{0}^{T} f(s, \phi(s), \psi(s)) d s$ belongs to $M^{2}\left(\Omega, \mathscr{F}_{T}, \mathbb{P} ; \mathbb{R}^{d}\right)$ :

$$
\begin{aligned}
& \mathbb{E}\left|\int_{0}^{T} f(s, \phi(s), \psi(s)) d s\right|^{2}=\mathbb{E}\left|\int_{0}^{T} \sqrt{p_{1}^{-1}(s) \alpha_{1}(s)} \frac{\sqrt{p_{1}(s)} f(s, \phi(s), \psi(s))}{\sqrt{\alpha_{1}(s)}} d s\right|^{2} \\
& \leq \mathbb{E}\left\{\left[\int_{0}^{T} p_{1}^{-1}(s) \alpha_{1}(s) d s\right]\left[\int_{0}^{T} \frac{p_{1}(s)|f(s, \phi(s), \psi(s))|^{2}}{\alpha_{1}(s)} d s\right]\right\} \\
& \leq \mathbb{E} \int_{0}^{T} \frac{p_{1}(s)|f(s, \phi(s), \psi(s))|^{2}}{\alpha_{1}(s)} d s \\
& =\mathbb{E} \int_{0}^{T} \frac{p_{1}(s)}{\alpha_{1}(s)} \mid f\left(s, \phi(s), \psi(s)-f(s, 0,0)+\left.f(s, 0,0)\right|^{2} d s\right. \\
& \leq \mathbb{E} \int_{0}^{T} \frac{p_{1}(s)}{\alpha_{1}(s)}\left[\mid f(s, \phi(s), \psi(s)-f(s, 0,0)|+| f(s, 0,0) \mid]^{2} d s\right. \\
& \leq \mathbb{E} \int_{0}^{T} \frac{p_{1}(s)}{\alpha_{1}(s)}\left[c_{1}(s)|\phi(s)|+c_{2}(s)|\psi(s)|+|f(s, 0,0)|\right]^{2} d s \\
& \leq \mathbb{E} \int_{0}^{T} \frac{p_{1}(s)}{\alpha_{1}(s)}\left[3 c_{1}^{2}(s)|\phi(s)|^{2}+3 c_{2}^{2}(s)|\psi(s)|^{2}+3|f(s, 0,0)|^{2}\right] d s \\
& =\mathbb{E} \int_{0}^{T} \frac{3 p_{1}(s)}{\beta_{1}} \frac{\beta_{1} c_{1}^{2}(s)}{\gamma(s)+\beta_{1} c_{1}^{2}(s)+\beta_{2} c_{2}^{2}(s)}|\phi(s)|^{2} d s+\frac{3 p_{1}(s)}{\beta_{2}} \frac{\beta_{2} c_{2}^{2}(s)}{\gamma(s)+\beta_{1} c_{1}^{2}(s)+\beta_{2} c_{2}^{2}(s)}|\psi(s)|^{2} d s \\
& +3 \mathbb{E} \int_{0}^{T} \frac{p_{1}(s)|f(s, 0,0)|^{2}}{\alpha_{1}(s)} d s \\
& \leq \frac{3}{\beta_{1}} \mathbb{E} \int_{0}^{T} p_{1}(s)|\phi(s)|^{2} d s+\frac{3}{\beta_{2}} \mathbb{E} \int_{0}^{T} p_{1}(s)|\psi(s)|^{2} d s+3 \mathbb{E} \int_{0}^{T} \frac{p_{1}(s)|f(s, 0,0)|^{2}}{\alpha_{1}(s)} d s<\infty
\end{aligned}
$$


Since $\xi \in \widehat{M}_{1}^{2}\left(\Omega, \mathscr{F}_{T}, \mathbb{P} ; \mathbb{R}^{d}\right)$ implies that $\xi \in M^{2}\left(\Omega, \mathscr{F}_{T}, \mathbb{P} ; \mathbb{R}^{d}\right)$, it follows from Lemma 2.1 of [34] that (8) has a unique solution pair $(y(\cdot), z(\cdot)) \in$ $M^{2}\left(0, T ; \mathbb{R}^{d}\right) \times M^{2}\left(0, T ; \mathbb{R}^{d \times k}\right)$. Moreover, since we proved that (9) is finite, it follows from Lemma 6.23 of [16] that in fact $(y(\cdot), z(\cdot)) \in \widehat{H}_{1}^{2}\left(0, T ; \mathbb{R}^{d}\right) \times$ $\widehat{M}_{1}^{2}\left(0, T ; \mathbb{R}^{d \times k}\right)$ and $\left[\sqrt{\alpha_{1}(\cdot)} y(\cdot)\right] \in \widehat{M}_{1}^{2}\left(0, T ; \mathbb{R}^{d}\right)$.

(ii) The proof follows closely that in [46] (pp. 307), and since it is short, we include it here for completeness. From the Burkholder-Davis-Gundy inequality (see, for example, Theorem 1.5.4 in [42]), there exists a constant $K$ such that

$$
\begin{array}{r}
\mathbb{E}\left[\sup _{t \in[0, T]}\left|\int_{0}^{t} p_{1}(s) y(s) z(s) d W(s)\right|\right] \leq K \mathbb{E}\left[\int_{0}^{T}\left|\sqrt{p_{1}(s)} y(s)\right|^{2}\left|\sqrt{p_{1}(s)} z(s)\right|^{2} d s\right]^{\frac{1}{2}} \\
\leq K \mathbb{E}\left[\left.\sup _{t \in[0, T]}\left|\sqrt{p_{1}(t)} y(t)\right|^{2} \int_{0}^{T} \sqrt{p_{1}(s)} z(s)\right|^{2} d s\right]^{\frac{1}{2}} \\
\leq \frac{K}{2} \mathbb{E}\left[\sup _{t \in[0, T]}\left|\sqrt{p_{1}(t)} y(t)\right|^{2}+\int_{0}^{T}\left|\sqrt{p_{1}(s)} z(s)\right|^{2} d s\right]<\infty,
\end{array}
$$

where the last step follows from the fact that $y(\cdot) \in \widehat{H}_{1}^{2}\left(0, T ; \mathbb{R}^{d}\right), z(\cdot) \in$ $\widehat{M}_{1}^{2}\left(0, T ; \mathbb{R}^{d \times k}\right)$, proved in part (i). The conclusion then follows from Corollary 7.22 of [26]. Since $\sup _{t \in[0, T]}\left|\sqrt{p_{1}(t)} y^{+}(t)\right|^{2} \leq \sup _{t \in[0, T]}\left|\sqrt{p_{1}(t)} y(t)\right|^{2}$, the conclusion follows even for $\int_{0}^{t} p_{1}(s) y^{+}(s) z(s) d W(s)$.

Lemma 2.2. Let $\phi(\cdot) \in \widehat{H}_{2}^{2}\left(0, T ; \mathbb{R}^{d}\right), \psi(\cdot) \in \widehat{M}_{2}^{2}\left(0, T ; \mathbb{R}^{d \times k}\right)$ be given, and assume that $\sqrt{\alpha_{2}(\cdot)} \phi(\cdot) \in \widehat{M}_{2}^{2}\left(0, T ; \mathbb{R}^{d}\right)$. If the pair $(f, \xi)$ satisfies the conditions A2, then:

(i) there exists a unique solution pair $(y(\cdot), z(\cdot)) \in \widehat{H}_{2}^{2}\left(0, T ; \mathbb{R}^{d}\right) \times \widehat{M}_{2}^{2}\left(0, T ; \mathbb{R}^{d \times k}\right)$ of equation

$$
y(t)=\xi+\int_{t}^{T} f(s, \phi(s), \psi(s)) d s-\int_{t}^{T} z(s) d W(s), \quad t \in[0, T],
$$

and $\sqrt{\alpha_{2}(\cdot)} y(\cdot) \in \widehat{M}_{2}^{2}\left(0, T ; \mathbb{R}^{d}\right)$.

\footnotetext{
${ }^{3}$ Note that the results in Lemma 6.2 of [16] is valid for any $\alpha(t)$ (in the notion of [16]).
} 
(ii) if $y^{+}(t) \equiv \mathbb{1}_{[y(t)>0]} y(t)$, the processes

$$
\int_{t}^{T} p_{2}(s) y(s) z(s) d W(s) \text { and } \int_{t}^{T} p_{2}(s) y^{+}(s) z(s) d W(s),
$$

are martingales.

Proof. The proof of part (ii) is the same as the proof of part (ii) of the previous lemma. We thus focus on part (i). We have

$$
\begin{aligned}
& \mathbb{E}\left|\int_{0}^{T} f(s, \phi(s), \psi(s)) d s\right|^{2} \\
\leq & \mathbb{E} \int_{0}^{T} \frac{p_{2}(s)}{\alpha_{2}(s)}\left[3 c_{1}^{2}(s)|\phi(s)|^{2}+3 c_{2}^{2}(s)|\psi(s)|^{2}+3|f(s, 0,0)|^{2}\right] d s \\
\leq & \mathbb{E} \int_{0}^{T} \frac{3 p_{2}(s)}{{\overline{\beta_{1}}}^{2}} \overline{\overline{\gamma(s)}+\overline{\beta_{1}} c_{1}(s)+\overline{\beta_{2}} c_{2}^{2}(s)}\left(\overline{\gamma(s)}+\overline{\beta_{1}} c_{1}(s)+\overline{\beta_{2}} c_{2}^{2}(s)\right)|\phi(s)|^{2} d s \\
& +\mathbb{E}_{0}^{T} \frac{3 p_{2}(s)}{\overline{\beta_{2}}} \overline{\overline{\gamma(s)}+\overline{\beta_{1}} c_{1}(s)+\overline{\beta_{2}} c_{2}^{2}(s)}|\psi(s)|^{2} d s+3 \mathbb{E} \int_{0}^{T} \frac{p_{2}(s)|f(s, 0,0)|^{2}}{\alpha_{2}(s)} d s \\
\leq & \frac{3}{{\overline{\beta_{1}}}^{2}} \mathbb{E} \int_{0}^{T} p_{2}(s) \alpha_{2}(s)|\phi(s)|^{2} d s+\frac{3}{\overline{\beta_{2}}} \mathbb{E} \int_{0}^{T} p_{2}(s)|\psi(s)|^{2} d s+3 \mathbb{E} \int_{0}^{T} \frac{p_{2}(s)|f(s, 0,0)|^{2}}{\alpha_{2}(s)} d s \\
< & \infty
\end{aligned}
$$

The rest of the proof is the same as in the proof of part (i) of the previous lemma.

Lemma 2.3. (i) Let $\phi(\cdot) \in \widehat{H}_{1}^{2}\left(0, T ; \mathbb{R}^{d}\right)$ be given. If the pair $(f, \xi)$ satisfies conditions $A 1$, then there exists a unique solution pair $(y(\cdot), z(\cdot)) \in$ $\widehat{H}_{1}^{2}\left(0, T ; \mathbb{R}^{d}\right) \times \widehat{M}_{1}^{2}\left(0, T ; \mathbb{R}^{d \times k}\right)$ of equation

$$
y(t)=\xi+\int_{t}^{T} f(s, \phi(s), z(s)) d s-\int_{t}^{T} z(s) d W(s), \quad t \in[0, T],
$$


and $\sqrt{\alpha_{1}(\cdot)} y(\cdot) \in \widehat{M}_{1}^{2}\left(0, T ; \mathbb{R}^{d}\right)$

(ii) Let $\phi(\cdot) \in \widehat{H}_{2}^{2}\left(0, T ; \mathbb{R}^{d}\right)$ be given. If the pair $(f, \xi)$ satisfies conditions A2, then there exists a unique solution pair $(y(\cdot), z(\cdot)) \in \widehat{H}_{2}^{2}\left(0, T ; \mathbb{R}^{d}\right) \times$ $\widehat{M}_{2}^{2}\left(0, T ; \mathbb{R}^{d \times k}\right)$ of equation

$$
y(t)=\xi+\int_{t}^{T} f(s, \phi(s), z(s)) d s-\int_{t}^{T} z(s) d W(s), \quad t \in[0, T],
$$

and $\sqrt{\alpha_{2}(\cdot)} y(\cdot) \in \widehat{M}_{2}^{2}\left(0, T ; \mathbb{R}^{d}\right)$

Proof. (i) (Uniqueness) Let $\left(y_{1}(\cdot), z_{1}(\cdot)\right)$ and $\left(y_{2}(\cdot), z_{2}(\cdot)\right)$ be two solution pairs of (11) with the claimed properties. Then

$$
\begin{aligned}
& -d p_{1}(t)\left|y_{1}(t)-y_{2}(t)\right|^{2} \\
= & \left\{-\alpha_{1}(t) p_{1}(t)\left|y_{1}(t)-y_{2}(t)\right|^{2}+2 p_{1}(t)\left(y_{1}(t)-y_{2}(t)\right)^{\prime}\left[f\left(t, \phi(t), z_{1}(t)\right)-f\left(t, \phi(t), z_{2}(t)\right)\right]\right. \\
& \left.-p_{1}(t)\left|z_{1}(t)-z_{2}(t)\right|^{2}\right\} d t-2 p_{1}(t)\left(y_{1}(t)-y_{2}(t)\right)^{\prime}\left(z_{1}(t)-z_{2}(t)\right) d W(t)
\end{aligned}
$$


By using the Lipschitz property of $f$, we have

$$
\begin{aligned}
& -d p_{1}(t)\left|y_{1}(t)-y_{2}(t)\right|^{2} \\
\leq & {\left[-\alpha_{1}(t) p_{1}(t)\left|y_{1}(t)-y_{2}(t)\right|^{2}-p_{1}(t)\left|z_{1}(t)-z_{2}(t)\right|^{2}\right] d t-2 p_{1}(t)\left(y_{1}(t)-y_{2}(t)\right)^{\prime}\left(z_{1}(t)-z_{2}(t)\right) d W(t) } \\
& +2 p_{1}(t)\left|y_{1}(t)-y_{2}(t)\right|\left|f\left(t, \phi(t), z_{1}(t)\right)-f\left(t, \phi(t), z_{2}(t)\right)\right| d t \\
\leq & {\left[-\alpha_{1}(t) p_{1}(t)\left|y_{1}(t)-y_{2}(t)\right|^{2}-p_{1}(t)\left|z_{1}(t)-z_{2}(t)\right|^{2}\right] d t-2 p_{1}(t)\left(y_{1}(t)-y_{2}(t)\right)^{\prime}\left(z_{1}(t)-z_{2}(t)\right) d W(t) } \\
& +2 p_{1}(t) c_{2}(t)\left|y_{1}(t)-y_{2}(t)\right|\left|z_{1}(t)-z_{2}(t)\right| d t \\
\leq & {\left[-\alpha_{1}(t) p_{1}(t)\left|y_{1}(t)-y_{2}(t)\right|^{2}-p_{1}(t)\left|z_{1}(t)-z_{2}(t)\right|^{2}\right] d t-2 p_{1}(t)\left(y_{1}(t)-y_{2}(t)\right)^{\prime}\left(z_{1}(t)-z_{2}(t)\right) d W(t) } \\
& +\beta_{2} c_{2}^{2}(t) p_{1}(t)\left|y_{1}(t)-y_{2}(t)\right|^{2} d t+\beta_{2}^{-1} p_{1}(t)\left|z_{1}(t)-z_{2}(t)\right|^{2} d t \\
\leq & -2 p_{1}(t)\left(y_{1}(t)-y_{2}(t)\right)^{\prime}\left(z_{1}(t)-z_{2}(t)\right) d W(t),
\end{aligned}
$$

which in integral form becomes

$$
p_{1}(t)\left|y_{1}(t)-y_{2}(t)\right|^{2} \leq \int_{t}^{T}-2 p_{1}(s)\left(y_{1}(s)-y_{2}(s)\right)^{\prime}\left(z_{1}(s)-z_{2}(s)\right) d W(s) \text {. }
$$

The stochastic integral in (13) is a local martingale that is clearly lower bounded by zero, and is thus a supermartingale (see, for example, Theorem 7.23 of [26]). Taking the expectation of both sides of (13) results in

$\mathbb{E}\left[p_{1}(t)\left|y_{1}(t)-y_{2}(t)\right|^{2}\right] \leq-\mathbb{E}\left[\int_{t}^{T} 2 p_{1}(s)\left(y_{1}(s)-y_{2}(s)\right)^{\prime}\left(z_{1}(s)-z_{2}(s)\right) d W(s)\right] \leq 0$.

Since $p_{1}(t)>0$, it follows that $y_{1}(t)=y_{2}(t), \forall t \in[0, T]$, a.s., which proves the uniqueness of $y(\cdot)$. Due to this fact, the integral form of (12) becomes

$$
0=\int_{t}^{T} p_{1}(s)\left|z_{1}(s)-z_{2}(s)\right|^{2} d s
$$

which implies that $z_{1}(t)=z_{2}(t)$ for a.e. $t \in[0, T]$, and thus proves the uniqueness of $z(\cdot)$. 
(Existence) Let $z_{0}(t) \equiv 0, \forall t \in[0, T]$, and for $n \geq 1$ consider the following sequence of equations:

$$
y_{n}(t)=\xi+\int_{t}^{T} f\left(s, \phi(s), z_{n-1}(s)\right) d s-\int_{t}^{T} z_{n}(s) d W(s), \quad t \in[0, T] .
$$

From Lemma 2.1 we know that these equations have unique solution pairs $\left\{\left(y_{n}(\cdot), z_{n}(\cdot)\right) \in \widehat{H}_{1}^{2}\left(0, T ; \mathbb{R}^{d}\right) \times \widehat{M}_{1}^{2}\left(0, T ; \mathbb{R}^{d \times k}\right)\right\}_{n \geq 1}$, for which it also holds that $\left\{\sqrt{\alpha_{1}(\cdot)} y_{n}(\cdot) \in \widehat{M}_{1}^{2}\left(0, T ; \mathbb{R}^{d}\right)\right\}_{n \geq 1}$. Similarly to the proof of uniqueness, 
we have

$$
\begin{aligned}
& -d p_{1}(t)\left|y_{n+1}(t)-y_{n}(t)\right|^{2} \\
& =\left\{-\alpha_{1}(t) p_{1}(t)\left|y_{n+1}(t)-y_{n}(t)\right|^{2}+2 p_{1}(t)\left(y_{n+1}(t)-y_{n}(t)\right)^{\prime}\left[f\left(t, \phi(t), z_{n}(t)\right)-f\left(t, \phi(t), z_{n-1}(t)\right)\right]\right. \\
& \left.-p_{1}(t)\left|z_{n+1}(t)-z_{n}(t)\right|^{2}\right\} d t-2 p_{1}(t)\left(y_{n+1}(t)-y_{n}(t)\right)^{\prime}\left(z_{n+1}(t)-z_{n}(t)\right) d W(t) \\
& \leq\left[-\alpha_{1}(t) p_{1}(t)\left|y_{n+1}(t)-y_{n}(t)\right|^{2}-p_{1}(t)\left|z_{n+1}(t)-z_{n}(t)\right|^{2}\right] d t \\
& -2 p_{1}(t)\left(y_{n+1}(t)-y_{n}(t)\right)^{\prime}\left(z_{n+1}(t)-z_{n}(t)\right) d W(t) \\
& +2 p_{1}(t)\left|y_{n+1}(t)-y_{n}(t)\right|\left|f\left(t, \phi(t), z_{n}(t)\right)-f\left(t, \phi(t), z_{n-1}(t)\right)\right| d t \\
& \leq\left[-\alpha_{1}(t) p_{1}(t)\left|y_{n+1}(t)-y_{n}(t)\right|^{2}-p_{1}(t)\left|z_{n+1}(t)-z_{n}(t)\right|^{2}\right] d t \\
& -2 p_{1}(t)\left(y_{n+1}(t)-y_{n}(t)\right)^{\prime}\left(z_{n+1}(t)-z_{n}(t)\right) d W(t) \\
& +2 p_{1}(t) c_{2}(t)\left|y_{n+1}(t)-y_{n}(t)\right|\left|z_{n}(t)-z_{n-1}(t)\right| d t \\
& \leq\left[-\alpha_{1}(t) p_{1}(t)\left|y_{n+1}(t)-y_{n}(t)\right|^{2}-p_{1}(t)\left|z_{n+1}(t)-z_{n}(t)\right|^{2}\right] d t \\
& -2 p_{1}(t)\left(y_{n+1}(t)-y_{n}(t)\right)^{\prime}\left(z_{n+1}(t)-z_{n}(t)\right) d W(t) \\
& +\beta_{2} c_{2}^{2}(t) p_{1}(t)\left|y_{n+1}(t)-y_{n}(t)\right|^{2} d t+\beta_{2}^{-1} p_{1}(t)\left|z_{n}(t)-z_{n-1}(t)\right|^{2} d t \\
& \leq\left[-p_{1}(t)\left|z_{n+1}(t)-z_{n}(t)\right|^{2}+\beta_{2}^{-1} p_{1}(t)\left|z_{n}(t)-z_{n-1}(t)\right|^{2}\right] d t \\
& -2 p_{1}(t)\left(y_{n+1}(t)-y_{n}(t)\right)^{\prime}\left(z_{n+1}(t)-z_{n}(t)\right) d W(t),
\end{aligned}
$$


which in integral form becomes

$$
\begin{aligned}
& p_{1}(t)\left|y_{n+1}(t)-y_{n}(t)\right|^{2}+\int_{t}^{T} p_{1}(s)\left|z_{n+1}(s)-z_{n}(s)\right|^{2} d s \\
& \left.\leq \beta_{2}^{-1} \int_{t}^{T} p_{1}(s)\left|z_{n}(s)-z_{n-1}(s)\right|^{2}\right] d s-\int_{t}^{T} 2 p_{1}(s)\left(y_{n+1}(s)-y_{n}(s)\right)^{\prime}\left(z_{n+1}(s)-z_{n}(s)\right) d W(s) .
\end{aligned}
$$

From Lemma 2.1(ii), it is clear that the stochastic integral on the right hand side is a martingale. Taking the expected values of both sides gives

$\left.\mathbb{E}\left[p_{1}(t)\left|y_{n+1}(t)-y_{n}(t)\right|^{2}\right]+\mathbb{E} \int_{t}^{T} p_{1}(s)\left|z_{n+1}(s)-z_{n}(s)\right|^{2} d s \leq \beta_{2}^{-1} \mathbb{E} \int_{t}^{T} p_{1}(s)\left|z_{n}(s)-z_{n-1}(s)\right|^{2}\right] d s$.

Let us define $\eta_{n}(t) \equiv \mathbb{E} \int_{t}^{T} p_{1}(s)\left|y_{n}(s)-y_{n-1}(s)\right|^{2} d s$ and $\mu_{n}(t) \equiv \mathbb{E} \int_{t}^{T} p_{1}(s) \mid z_{n}(s)-$ $\left.z_{n-1}(s)\right|^{2} d s$. Using the same argument as in the last part of the proof of Proposition 2.2 in [34], we obtain $\eta_{n+1}(0) \leq \beta_{2}^{-n} \mathbb{E} \int_{0}^{T} p_{1}(s)\left|z_{1}(s)\right|^{2} d s$ and $\mu_{n}(0) \leq \beta_{2}^{-n} \mu_{1}(0)$. Since the right-hand sides of these two inequalities decrease with $n$, it follows that $\left\{y_{n}\right\}_{n \geq 1}$ is a Cauchy sequence in $\widehat{M}_{1}^{2}\left(0, T ; \mathbb{R}^{d}\right)$, and $\left\{z_{n}\right\}_{n \geq 1}$ is a Cauchy sequence in $\widehat{M}_{1}^{2}\left(0, T ; \mathbb{R}^{d \times k}\right)$. Moreover, this also implies that $\left\{\sqrt{\alpha_{1}} y_{n}\right\}_{n \geq 1}$ is a Cauchy sequence in $\widehat{M}_{1}^{2}\left(0, T ; \mathbb{R}^{d}\right)$. Hence, the limiting processes $y^{*}=\lim _{n \rightarrow \infty} y_{n}$ and $z^{*}=\lim _{n \rightarrow \infty} z_{n}$ are the solution pair of (11). In addition, when such a pair of processes is substituted in (11), then (11) becomes an example of (18) with $\psi(\cdot)=z^{*}(\cdot)$. Therefore, Lemma 2.1 applies, and we have that $y^{*}(\cdot) \in \widehat{H}_{1}^{2}\left(0, T ; \mathbb{R}^{d \times k}\right)$.

(ii) Due to Lemma 2.2, the proof in this case is identical to the proof of part (i) (with an obvious change of notation), and is thus omitted.

Theorem 2.1. (i) If the pair $(f, \xi)$ satisfies conditions A1, then equation (1) has a unique solution pair $(y(\cdot), z(\cdot)) \in \widehat{H}_{1}^{2}\left(0, T ; \mathbb{R}^{d}\right) \times \widehat{M}_{1}^{2}\left(0, T ; \mathbb{R}^{d \times k}\right)$, and $\sqrt{\alpha_{1}(\cdot)} y(\cdot) \in \widehat{M}_{1}^{2}\left(0, T ; \mathbb{R}^{d}\right)$.

(ii) If the pair $(f, \xi)$ satisfies conditions A2, then equation (1) has a unique solution pair $(y(\cdot), z(\cdot)) \in \widehat{H}_{2}^{2}\left(0, T ; \mathbb{R}^{d}\right) \times \widehat{M}_{2}^{2}\left(0, T ; \mathbb{R}^{d \times k}\right)$, and $\sqrt{\alpha_{2}(\cdot)} y(\cdot) \in$ $\widehat{M}_{2}^{2}\left(0, T ; \mathbb{R}^{d}\right)$. 
Proof. (i) (Uniqueness) Let $\left(y_{1}(\cdot), z_{1}(\cdot)\right)$ and $\left(y_{2}(\cdot), z_{2}(\cdot)\right)$ be two solution pairs of (1) with the claimed properties. Then we have

$$
\begin{aligned}
& -d p_{1}(t)\left|y_{1}(t)-y_{2}(t)\right|^{2} \\
= & \left\{-\alpha_{1}(t) p_{1}(t)\left|y_{1}(t)-y_{2}(t)\right|^{2}+2 p_{1}(t)\left(y_{1}(t)-y_{2}(t)\right)^{\prime}\left[f\left(t, y_{1}(t), z_{1}(t)\right)-f\left(t, y_{2}(t), z_{2}(t)\right]\right.\right. \\
& \left.-p_{1}(t)\left|z_{1}(t)-z_{2}(t)\right|^{2}\right\} d t-2 p_{1}(t)\left(y_{1}(t)-y_{2}(t)\right)^{\prime}\left(z_{1}(t)-z_{2}(t)\right) d W(t) \\
\leq & {\left[-\alpha_{1}(t) p_{1}(t)\left|y_{1}(t)-y_{2}(t)\right|^{2}-p_{1}(t)\left|z_{1}(t)-z_{2}(t)\right|^{2}\right] d t-2 p_{1}(t)\left(y_{1}(t)-y_{2}(t)\right)^{\prime}\left(z_{1}(t)-z_{2}(t)\right) d W(t) } \\
& +2 p_{1}(t)\left|y_{1}(t)-y_{2}(t)\right|\left|f\left(t, y_{1}(t), z_{1}(t)\right)-f\left(t, y_{2}(t), z_{2}(t)\right)\right| d t \\
\leq & \left.-\alpha_{1}(t) p_{1}(t)\left|y_{1}(t)-y_{2}(t)\right|^{2}-p_{1}(t)\left|z_{1}(t)-z_{2}(t)\right|^{2}\right] d t-2 p_{1}(t)\left(y_{1}(t)-y_{2}(t)\right)^{\prime}\left(z_{1}(t)-z_{2}(t)\right) d W(t) \\
& +2 p_{1}(t)\left|y_{1}(t)-y_{2}(t)\right|\left[c_{1}(t)\left|y_{1}(t)-y_{2}(t)\right|+c_{2}(t)\left|z_{1}(t)-z_{2}(t)\right|\right] d t \\
\leq & \left.-\alpha_{1}(t) p_{1}(t)\left|y_{1}(t)-y_{2}(t)\right|^{2}-p_{1}(t)\left|z_{1}(t)-z_{2}(t)\right|^{2}\right] d t-2 p_{1}(t)\left(y_{1}(t)-y_{2}(t)\right)^{\prime}\left(z_{1}(t)-z_{2}(t)\right) d W(t) \\
& +\beta_{1} c_{1}^{2} p_{1}(t)\left|y_{1}(t)-y_{2}(t)\right|^{2} d t+\beta_{1}^{-1} p_{1}(t)\left|y_{1}(t)-y_{2}(t)\right|^{2} d t \\
& -2 p_{1}(t)\left(y_{1}(t)-y_{2}(t)\right)^{\prime}\left(z_{1}(t)-z_{2}(t)\right) d W(t) \\
& +\beta_{2} c_{2}^{2} p_{1}(t)\left|y_{1}(t)-y_{2}(t)\right|^{2} d t+\beta_{2}^{-1} p_{1}(t)\left|z_{1}(t)-z_{2}(t)\right|^{2} d t \\
\leq & {\left[\beta_{1}^{-1} p_{1}(t)\left|y_{1}(t)-y_{2}(t)\right|^{2}+\left(\beta_{2}^{-1}-1\right) p_{1}(t)\left|z_{1}(t)-z_{2}(t)\right|^{2}\right] d t } \\
& \\
& \\
& \\
&
\end{aligned}
$$

With the help of Lemma 2.1 (ii) and the Gronwall's lemma, the conclusion follows similarly to the proof of uniqueness in Lemma 2.3 .

(Existence) Let $y_{0}(t) \equiv 0, \forall t \in[0, T]$, and for $n \geq 1$ consider the sequence 
of equations:

$$
y_{n}(t)=\xi+\int_{t}^{T} f\left(s, y_{n-1}(s), z_{n}(s)\right) d s-\int_{t}^{T} z_{n}(s) d W(s), \quad t \in[0, T] .
$$

From Lemma 2.3 we know that these equations have unique solution pairs $\left\{\left(y_{n}(\cdot), z_{n}(\cdot)\right) \in \widehat{H}_{1}^{2}\left(0, T ; \mathbb{R}^{d}\right) \times \widehat{M}_{1}^{2}\left(0, T ; \mathbb{R}^{d \times k}\right)\right\}_{n \geq 1}$. Then

$$
\begin{aligned}
& -d p_{1}(t)\left|y_{n+1}(t)-y_{n}(t)\right|^{2} \\
= & \left\{\alpha_{1}(t) p_{1}(t)\left|y_{n+1}(t)-y_{n}(t)\right|^{2}+2 p_{1}(t)\left(y_{n+1}(t)-y_{n}(t)\right)^{\prime}\left[f\left(t, y_{n}(t), z_{n+1}(t)\right)-f\left(t, y_{n-1}(t), z_{n}(t)\right)\right]\right. \\
& \left.-p_{1}(t)\left|z_{n+1}(t)-z_{n}(t)\right|^{2}\right\} d t-2 p_{1}(t)\left(y_{n+1}(t)-y_{n}(t)\right)^{\prime}\left(z_{n+1}(t)-z_{n}(t)\right) d W(t) \\
\leq & {\left[-\alpha_{1}(t) p_{1}(t)\left|y_{n+1}(t)-y_{n}(t)\right|^{2}-p_{1}(t)\left|z_{n+1}(t)-z_{n}(t)\right|^{2}\right] d t } \\
& -2 p_{1}(t)\left(y_{n+1}(t)-y_{n}(t)\right)^{\prime}\left(z_{n+1}(t)-z_{n}(t)\right) d W(t) \\
& +2 p_{1}(t)\left|y_{n+1}(t)-y_{n}(t)\right|\left|f\left(t, y_{n}(t), z_{n+1}(t)\right)-f\left(t, y_{n-1}(t), z_{n}(t)\right)\right| d t \\
\leq & \left.-\alpha_{1}(t) p_{1}(t)\left|y_{n+1}(t)-y_{n}(t)\right|^{2}-p_{1}(t)\left|z_{n+1}(t)-z_{n}(t)\right|^{2}\right] d t \\
& -2 p_{1}(t)\left(y_{n+1}(t)-y_{n}(t)\right)^{\prime}\left(z_{n+1}(t)-z_{n}(t)\right) d W(t) \\
& +\beta_{2} c_{2}^{2}(t) p_{1}(t)\left|y_{n+1}(t)-y_{n}(t)\right|^{2} d t+\beta_{2}^{-1} p_{1}(t)\left|z_{n+1}(t)-z_{n}(t)\right|^{2} d t \\
& +2 p_{1}(t)\left|y_{n+1}(t)-y_{n}(t)\right|\left[c_{1}(t)\left|y_{n}(t)-y_{n-1}(t)\right|+c_{2}(t)\left|z_{n+1}(t)-z_{n}(t)\right|\right] d t \\
& + \\
& \left.-\alpha_{1}(t) p_{1}^{2}(t)\left|p_{n+1}(t)-y_{n}(t)\right|^{2}-p_{1}(t)\left|z_{n+1}(t)-z_{n}(t)\right|^{2}\right] d t \\
& \\
& \\
& \\
& \\
& \\
& \\
&
\end{aligned}
$$




$$
\begin{aligned}
\leq & \beta_{1}^{-1} p_{1}(t)\left|y_{n}(t)-y_{n-1}(t)\right|^{2} d t+\left(\beta_{2}^{-1}-1\right) p_{1}(t)\left|z_{n+1}(t)-z_{n}(t)\right|^{2} d t \\
& -2 p_{1}(t)\left(y_{n+1}(t)-y_{n}(t)\right)^{\prime}\left(z_{n+1}(t)-z_{n}(t)\right) d W(t) .
\end{aligned}
$$

Due to Lemma 2.1 (ii), the expectation of the integral-form of this inequity becomes

$$
\begin{aligned}
\mathbb{E}\left[p_{1}(t)\left|y_{n+1}(t)-y_{n}(t)\right|^{2}\right] \leq & \mathbb{E} \int_{t}^{T} \beta_{1}^{-1} p_{1}(s)\left|y_{n}(s)-y_{n-1}(s)\right|^{2} d s \\
& +\mathbb{E} \int_{t}^{T}\left(\beta_{2}^{-1}-1\right) p_{1}(s)\left|z_{n+1}(s)-z_{n}(s)\right|^{2} d s
\end{aligned}
$$

Using the notation $\nu_{n+1}(t) \equiv \mathbb{E} \int_{t}^{T} p_{1}(t)\left|y_{n+1}(s)-y_{n}(s)\right|^{2} d s$, and similarly to the last part of the proof of Theorem 3.1 of [34], we obtain $\nu_{n+1}(0) \leq$ $\beta_{1}^{-n} \frac{1}{n !} \nu_{1}(0)$. Since the sum of the right-hand side of this inequality converges, we conclude, together with (16), that $\left\{y_{n}\right\}$ is a Cauchy sequence in $\widehat{M}_{1}^{2}\left(0, T ; \mathbb{R}^{d}\right)$, and $\left\{z_{n}\right\}$ is a Cauchy sequence in $\widehat{M}_{1}^{2}\left(0, T ; \mathbb{R}^{d \times k}\right)$. Moreover, this also implies that $\left\{\sqrt{\alpha} y_{n}\right\}_{n \geq 1}$ is a Cauchy sequence in $\widehat{M}_{1}^{2}\left(0, T ; \mathbb{R}^{d}\right)$. Thus the limiting processes $y^{*}=\lim _{n \rightarrow \infty} y_{n}$ and $z^{*}=\lim _{n \rightarrow \infty} z_{n}$ are the solution pair to (10). In addition, when such a pair of processes is substituted in (11), then (10) becomes an example of (8) with $\phi(\cdot)=y^{*}(\cdot)$ and $\psi(\cdot)=z^{*}(\cdot)$. Therefore, Lemma 2.1 applies, and we have that $y^{*}(\cdot) \in \widehat{H}_{1}^{2}\left(0, T ; \mathbb{R}^{d \times k}\right)$.

(ii) (Uniqueness) Let $\left(y_{1}(\cdot), z_{1}(\cdot)\right)$ and $\left(y_{2}(\cdot), z_{2}(\cdot)\right)$ be two solution pairs of (1) with the claimed properties. Similarly to the proof of uniqueness for 
part (i), we have

$$
\begin{aligned}
& -d p_{2}(t)\left|y_{1}(t)-y_{2}(t)\right|^{2} \\
\leq & {\left[-\alpha_{2}(t) p_{2}(t)\left|y_{1}(t)-y_{2}(t)\right|^{2}-p_{2}(t)\left|z_{1}(t)-z_{2}(t)\right|^{2}\right] d t-2 p_{2}(t)\left(y_{1}(t)-y_{2}(t)\right)^{\prime}\left(z_{1}(t)-z_{2}(t)\right) d W(t) } \\
& +2 p_{2}(t)\left|y_{1}(t)-y_{2}(t)\right|\left[c_{1}(t)\left|y_{1}(t)-y_{2}(t)\right|+c_{2}(t)\left|z_{1}(t)-z_{2}(t)\right|\right] d t \\
\leq & {\left[-\alpha_{2}(t) p_{2}(t)\left|y_{1}(t)-y_{2}(t)\right|^{2}-p_{2}(t)\left|z_{1}(t)-z_{2}(t)\right|^{2}\right] d t-2 p_{2}(t)\left(y_{1}(t)-y_{2}(t)\right)^{\prime}\left(z_{1}(t)-z_{2}(t)\right) d W(t) } \\
& +2 c_{1} p_{2}(t)\left|y_{1}(t)-y_{2}(t)\right|^{2} d t+\overline{\beta_{2}} c_{2}^{2} p_{2}(t)\left|y_{1}(t)-y_{2}(t)\right|^{2} d t+\bar{\beta}_{2}-1 p_{2}(t)\left|z_{1}(t)-z_{2}(t)\right|^{2} d t \\
\leq & \left(\beta_{2}^{-1}-1\right) p_{2}(t)\left|z_{1}(t)-z_{2}(t)\right|^{2} d t-2 p_{2}(t)\left(y_{1}(t)-y_{2}(t)\right)^{\prime}\left(z_{1}(t)-z_{2}(t)\right) d W(t) \\
\leq & -2 p_{2}(t)\left(y_{1}(t)-y_{2}(t)\right)^{\prime}\left(z_{1}(t)-z_{2}(t)\right) d W(t) .
\end{aligned}
$$

Then the expectation of integral-form of this inequality becomes

$\mathbb{E}\left[p_{2}(t)\left|y_{1}(t)-y_{2}(t)\right|^{2}\right] \leq \mathbb{E}\left[\int_{t}^{T}-2 p_{2}(s)\left(y_{1}(s)-y_{2}(s)\right)^{\prime}\left(z_{1}(s)-z_{2}(s)\right) d W(s)\right]$.

Since the right-hand side is a martingale by Lemma 2.1 (ii), the conclusion follows.

(Existence) Let $y_{0}(t) \equiv 0, \forall t \in[0, T]$, and for $n \geq 1$ consider the sequence of equations:

$$
y_{n}(t)=\xi+\int_{t}^{T} f\left(s, y_{n-1}(s), z_{n}(s)\right) d s-\int_{t}^{T} z_{n}(s) d W(s), \quad t \in[0, T] .
$$

From Lemma 2.3 we know that these equations have unique solution pairs $\left\{\left(y_{n}(\cdot), z_{n}(\cdot)\right) \in \widehat{H}_{2}^{2}\left(0, T ; \mathbb{R}^{d}\right) \times \widehat{M}_{2}^{2}\left(0, T ; \mathbb{R}^{d \times k}\right)\right\}_{n \geq 1}$. By Lemma 6.2 of [16], we have following estimates:

$$
\begin{aligned}
& \mathbb{E}\left[\int_{0}^{T} p_{2}(t)\left|y_{n+1}(t)-y_{n}(t)\right|^{2} \alpha_{2}(t) d t\right] \\
\leq & 8 \mathbb{E}\left[\int_{0}^{T} p_{2}(t) \frac{\left|f\left(t, y_{n}(t), z_{n+1}(t)\right)-f\left(t, y_{n-1}(t), z_{n}(t)\right)\right|^{2}}{\alpha_{2}(t)} d t\right],
\end{aligned}
$$


and

$$
\begin{aligned}
& \mathbb{E}\left[\int_{0}^{T} p_{2}(t)\left|z_{n+1}(t)-z_{n}(t)\right|^{2} d t\right] \\
\leq & 45 \mathbb{E}\left[\int_{0}^{T} p_{2}(t) \frac{\left|f\left(t, y_{n}(t), z_{n+1}(t)\right)-f\left(t, y_{n-1}(t), z_{n}(t)\right)\right|^{2}}{\alpha_{2}(t)} d t\right] .
\end{aligned}
$$

By the Lipschitz condition, we have

$$
\begin{aligned}
& \mathbb{E}\left[\int_{0}^{T} p_{2}(t) \frac{\left|f\left(t, y_{n}(t), z_{n+1}(t)\right)-f\left(t, y_{n-1}(t), z_{n}(t)\right)\right|^{2}}{\alpha_{2}(t)} d t\right] \\
\leq & \mathbb{E} \int_{0}^{T} \frac{p_{2}(t)}{\alpha_{2}(t)}\left[c_{1}(t)\left|y_{n}(t)-y_{n-1}(t)\right|+c_{2}(t)\left|z_{n+1}(t)-z_{n}(t)\right|\right]^{2} d t \\
\leq & 2 \mathbb{E} \int_{0}^{T} \frac{p_{2}(t)}{\alpha_{2}(t)}\left[c_{1}^{2}(t)\left|y_{n}(t)-y_{n-1}(t)\right|^{2}+c_{2}^{2}(t)\left|z_{n+1}(t)-z_{n}(t)\right|^{2}\right] d t \\
\leq & 2 \mathbb{E} \int_{0}^{T} \frac{p_{2}(t)}{\overline{\beta_{1}}} \overline{\overline{\gamma(t)}+\overline{\beta_{1}} c_{1}(t)+\overline{\beta_{2}} c_{2}^{2}(t)}\left(\overline{\gamma(t)}+\overline{\beta_{1}} c_{1}(t)+\overline{\beta_{2}} c_{2}^{2}(t)\right)\left|y_{n}(t)-y_{n-1}(t)\right|^{2} d t \\
& \left.+2 \mathbb{E} \int_{0}^{T} \frac{p_{2}(t)}{\overline{\beta_{2}}} \overline{\overline{\gamma(t)}+\overline{\beta_{1}} c_{1}(t)+\overline{\beta_{2}} c_{2}^{2}(t)}\left|z_{n+1}(t)-z_{n}(t)\right|\right]^{2} d s \\
\leq & \left.\frac{2}{\bar{\beta}_{1}^{2}} \mathbb{E} \int_{0}^{T} p_{2}(t) \alpha_{2}(t)\left|y_{n}(t)-y_{n-1}(t)\right|^{2} d t+\frac{2}{\overline{\beta_{2}}} \mathbb{E} \int_{0}^{T} p_{2}(t)\left|z_{n+1}(t)-z_{n}(t)\right|\right]^{2} d t .
\end{aligned}
$$

Substituting it into (19), we have

$$
\begin{aligned}
& \mathbb{E}\left[\int_{0}^{T} p_{2}(t)\left|z_{n+1}(t)-z_{n}(t)\right|^{2} d t\right] \\
\leq & \left.\frac{90}{{\overline{\beta_{1}}}^{2}} \mathbb{E} \int_{0}^{T} p_{2}(t) \alpha_{2}(t)\left|y_{n}(t)-y_{n-1}(t)\right|^{2} d t+\frac{90}{\overline{\beta_{2}}} \mathbb{E} \int_{0}^{T} p_{2}(t)\left|z_{n+1}(t)-z_{n}(t)\right|\right]^{2} d t .
\end{aligned}
$$


Let $\overline{\beta_{2}}>90$. Then we have

$\mathbb{E}\left[\int_{0}^{T} p_{2}(t)\left|z_{n+1}(t)-z_{n}(t)\right|^{2} d t\right] \leq \frac{{\overline{\bar{\beta}_{1}}}^{2}}{\left(1-\frac{90}{\bar{\beta}_{2}}\right)} \mathbb{E} \int_{0}^{T} p_{2}(t) \alpha_{2}(t)\left|y_{n}(t)-y_{n-1}(t)\right|^{2} d t$.

Substituting it into (19), we obtain

$$
\begin{aligned}
& \mathbb{E}\left[\int_{0}^{T} p_{2}(t)\left|y_{n+1}(t)-y_{n}(t)\right|^{2} \alpha_{2}(t) d t\right] \\
& \left.\leq \frac{16}{{\overline{\beta_{1}}}^{2}} \mathbb{E} \int_{0}^{T} p_{2}(t) \alpha_{2}(t)\left|y_{n}(t)-y_{n-1}(t)\right|^{2} d t+\frac{16}{\overline{\beta_{2}}} \mathbb{E} \int_{0}^{T} p_{2}(t)\left|z_{n+1}(t)-z_{n}(t)\right|\right]^{2} d t \\
& \leq \frac{16}{{\overline{\beta_{1}}}^{2}} \mathbb{E} \int_{0}^{T} p_{2}(t) \alpha_{2}(t)\left|y_{n}(t)-y_{n-1}(t)\right|^{2} d t+\frac{16}{\overline{\beta_{2}}} \frac{\frac{90}{{\overline{\beta_{1}}}^{2}}}{\left(1-\frac{90}{\beta_{2}}\right)} \mathbb{E} \int_{0}^{T} p_{2}(t) \alpha_{2}(t)\left|y_{n}(t)-y_{n-1}(t)\right|^{2} d t \\
& =\left[\frac{16}{{\overline{\beta_{1}}}^{2}}+\frac{16}{\overline{\beta_{2}}} \frac{{\overline{\bar{\beta}_{1}}}^{2}}{\left(1-\frac{90}{\bar{\beta}_{2}}\right)}\right] \mathbb{E} \int_{0}^{T} p_{2}(t) \alpha_{2}(t)\left|y_{n}(t)-y_{n-1}(t)\right|^{2} d t \\
& \text { Let } \kappa=\left[\frac{16}{{\overline{\beta_{1}}}^{2}}+\frac{16}{\bar{\beta}_{2}} \frac{\frac{90}{{\overline{\beta_{1}}}^{2}}}{\left(1-\frac{90}{\bar{\beta}_{2}}\right)}\right] \text {, i.e. } \bar{\beta}_{1}>4 \text { and } \bar{\beta}_{2}>\frac{90 \bar{\beta}_{1}^{2}}{\bar{\beta}_{1}^{2}-16} \text {. Then the conclu- } \\
& \text { sion follows similarly to the proof of existence in Lemma } 2.3 \text {. }
\end{aligned}
$$

\subsection{Comparison theorem}

The following results generalise Peng's comparison theorem ([35], [36]) to equations with a possibly unbounded generator. Similarly to [35], [36], we assume that $d=1$. In addition to equation (11), let us consider two further equations

$$
\begin{aligned}
& \widehat{y}_{1}(t)=\widehat{\xi}_{1}+\int_{t}^{T}\left[\widehat{f}_{1}\left(s, \widehat{y}_{1}(s), \widehat{z}_{1}(s)\right)\right] d s-\int_{t}^{T} \widehat{z}_{1}(s) d W(s), \quad t \in[0, T], \\
& \widehat{y}_{2}(t)=\widehat{\xi}_{2}+\int_{t}^{T}\left[\widehat{f}_{2}\left(s, \widehat{y}_{2}(s), \widehat{z}_{2}(s)\right)\right] d s-\int_{t}^{T} \widehat{z}_{2}(s) d W(s), \quad t \in[0, T] .
\end{aligned}
$$


We assume that the pair $\left(\widehat{f}_{1}, \widehat{\xi}_{1}\right)$ satisfies conditions $\mathrm{A} 1$, whereas the pair $\left(\widehat{f}_{2}, \widehat{\xi}_{2}\right)$ satisfies conditions A2. Based on Theorem 2.1, this means that there exist unique solution pairs $\left(\widehat{y}_{1}(\cdot), \widehat{z}_{1}(\cdot)\right) \in \widehat{H}_{1}^{2}(0, T ; \mathbb{R}) \times \widehat{M}_{1}^{2}\left(0, T ; \mathbb{R}^{1 \times k}\right)$ and $\left(\widehat{y}_{2}(\cdot), \widehat{z}_{2}(\cdot)\right) \in \widehat{H}_{2}^{2}(0, T ; \mathbb{R}) \times \widehat{M}_{2}^{2}\left(0, T ; \mathbb{R}^{1 \times k}\right)$. The following differences will appear in the proof:

$$
\begin{aligned}
& Y_{1}(t) \equiv y(t)-\widehat{y}_{1}(t), \quad Z_{1}(t) \equiv z(t)-\widehat{z}_{1}(t), \\
& Y_{2}(t) \equiv y(t)-\widehat{y}_{2}(t), \quad Z_{2}(t) \equiv z(t)-\widehat{z}_{2}(t) .
\end{aligned}
$$

Theorem 2.2. (Comparison theorem) (i) If $\widehat{\xi}_{1} \geq \xi$ and $\widehat{f}_{1}(t, y, z) \geq f(t, y, z)$, a.s. $\forall(t, y, z) \in[0, T] \times \mathbb{R} \times \mathbb{R}^{1 \times k}$, then $\widehat{y}_{1}(t) \geq y(t), \forall t \in[0, T]$, a.s..

(ii) If $\widehat{\xi}_{2} \geq \xi$ and $\widehat{f}_{2}(t, y, z) \geq f(t, y, z)$, a.s. $\forall(t, y, z) \in[0, T] \times \mathbb{R} \times \mathbb{R}^{1 \times k}$, then $\widehat{y}_{2}(t) \geq y(t), \forall t \in[0, T]$, a.s..

Proof. (i) The equation of the difference $Y_{1}(t)$ is

$$
-d Y_{1}(t)=\left[f(t, y(t), z(t))-\widehat{f}_{1}\left(t, \widehat{y}_{1}(t), \widehat{z}_{1}(t)\right)\right] d t-Z_{1}(t) d W(t) .
$$

Denoting by $Y_{1}^{+}(t) \equiv \mathbb{1}_{\left[Y_{1}(t)>0\right]} Y_{1}(t)$, and using Tanaka-Meyer formula (see Theorem 6.1.2 in [38]), we obtain

$$
-d Y_{1}^{+}(t)=-\mathbb{1}_{\left[Y_{1}(t)>0\right]} d Y_{1}(t)-\frac{1}{2} d L(t),
$$

where $L(t)$ is the local time of $Y_{1}(\cdot)$ at 0 . Since $\int_{0}^{T}\left|Y_{1}(t)\right| d L(t)=0$, a.s. (see Proposition 6.1.3 in [38]), we have

$$
\begin{aligned}
-d\left[Y_{1}^{+}(t)\right]^{2} & =2 Y_{1}^{+}(t) \mathbb{1}_{\left[Y_{1}(t)>0\right]}\left[f(t, y(t), z(t))-\widehat{f}_{1}\left(t, \widehat{y}_{1}(t), \widehat{z}_{1}(t)\right)\right] d t \\
& -\mathbb{1}_{\left[Y_{1}(t)>0\right]} Z_{1}^{2}(t) d t-\mathbb{1}_{\left[Y_{1}(t)>0\right]} 2 Y_{1}^{+}(t) Z_{1}(t) d W(t) .
\end{aligned}
$$


Using Itô formula, we obtain

$$
\begin{aligned}
& -d p_{1}(t)\left[Y_{1}^{+}(t)\right]^{2} \\
= & -\alpha_{1}(t) p_{1}(t)\left[Y_{1}^{+}(t)\right]^{2} d t+2 p_{1}(t) Y_{1}^{+}(t) \mathbb{1}_{\left[Y_{1}(t)>0\right]}\left[f(t, y(t), z(t))-\widehat{f}_{1}\left(t, \widehat{y}_{1}(t), \widehat{z}_{1}(t)\right)\right] d t \\
& -\mathbb{1}_{\left[Y_{1}(t)>0\right]} p_{1}(t) Z_{1}^{2}(t) d t-2 p_{1}(t) Y_{1}^{+}(t) Z_{1}(t) d W(t) \\
\leq & -\alpha_{1}(t) p_{1}(t)\left[Y_{1}^{+}(t)\right]^{2} d t+2 p_{1}(t) Y_{1}^{+}(t) \mathbb{1}_{\left[Y_{1}(t)>0\right]}\left[f(t, y(t), z(t))-\widehat{f}_{1}(t, y(t), z(t))\right. \\
& \left.+\widehat{f}_{1}(t, y(t), z(t))-\widehat{f}_{1}\left(t, \widehat{y}_{1}(t), \widehat{z}_{1}(t)\right)\right] d t \\
& -\mathbb{1}_{\left[Y_{1}(t)>0\right]} p_{1}(t) Z_{1}^{2}(t) d t-2 p_{1}(t) Y_{1}^{+}(t) Z_{1}(t) d W(t) \\
\leq & -\alpha_{1}(t) p_{1}(t)\left[Y_{1}^{+}(t)\right]^{2}+2 p_{1}(t) Y_{1}^{+}(t) \mathbb{1}_{\left[Y_{1}(t)>0\right]}\left[f(t, y(t), z(t))-\widehat{f}_{1}(t, y(t), z(t))\right] \\
& \left.-\mathbb{1}_{\left[Y_{1}(t)>0\right]} p_{1}(t) Z_{1}^{2}(t)\right] d t-\mathbb{1}_{\left[Y_{1}(t)>0\right]} 2 p_{1}(t) Y_{1}^{+}(t) Z_{1}(t) d W(t)+\beta_{1} p_{1}(t) c_{1}^{2}(t)\left[Y_{1}^{+}(t)\right]^{2} d t \\
& +\beta_{1}^{-1} p_{1}(t)\left[Y_{1}^{+}(t)\right]^{2} d t+\beta_{2} c_{2}^{2}(t) p_{1}(t)\left[Y_{1}^{+}(t)\right]^{2} d t+\beta_{2}^{-1} \mathbb{1}_{\left[Y_{1}(t)>0\right]} p_{1}(t) Z_{1}^{2}(t) d t \\
\leq & \beta_{1}^{-1} p_{1}(t)\left[Y_{1}^{+}(t)\right]^{2} d t-2 p_{1}(t) Y_{1}^{+}(t) Z_{1}(t) d W(t),
\end{aligned}
$$

which in integral form becomes

$$
p_{1}(t)\left[Y_{1}^{+}(t)\right]^{2} \leq \int_{t}^{T} \beta_{1}^{-1} p_{1}(s)\left[Y_{1}^{+}(s)\right]^{2} d s-\int_{t}^{T} 2 p_{1}(s) Y_{1}^{+}(s) Z_{1}(s) d W(s) .
$$

The stochastic integral on the right-hand side is a martingale due to Lemma 2.1 (ii). Therefore,

$$
\mathbb{E}\left[p_{1}(t)\left[Y_{1}^{+}(t)\right]^{2}\right] \leq \mathbb{E} \int_{t}^{T} \beta_{1}^{-1} p_{1}(s)\left[Y_{1}^{+}(s)\right]^{2} d s
$$

and the conclusion follows from Gronwall's lemma. 
(ii) In a similar way to the proof of part (i), we have

$$
\begin{aligned}
& -d p_{2}(t)\left[Y_{2}^{+}(t)\right]^{2} \\
= & -\alpha_{2}(t) p_{2}(t)\left[Y_{2}^{+}(t)\right]^{2} d t+2 p_{2}(t) Y_{2}^{+}(t) \mathbb{1}_{\left[Y_{2}(t)>0\right]}\left[f(t, y(t), z(t))-\widehat{f}_{2}\left(t, \widehat{y}_{2}(t), \widehat{z}_{2}(t)\right)\right] d t \\
& -\mathbb{1}_{\left[Y_{2}(t)>0\right]} p_{2}(t) Z_{2}^{2}(t) d t-2 p_{2}(t) Y_{2}^{+}(t) Z_{2}(t) d W(t) \\
\leq & -\alpha_{2}(t) p_{2}(t)\left[Y_{2}^{+}(t)\right]^{2} d t+2 p_{2}(t) Y_{2}^{+}(t) \mathbb{1}_{\left[Y_{2}(t)>0\right]}\left[f(t, y(t), z(t))-\widehat{f}_{2}(t, y(t), z(t))\right. \\
& \left.+\widehat{f}_{2}(t, y(t), z(t))-\widehat{f}_{2}\left(t, \widehat{y}_{2}(t), \widehat{z}_{2}(t)\right)\right] d t \\
& -\mathbb{1}_{\left[Y_{2}(t)>0\right]} p_{2}(t) Z_{2}^{2}(t) d t-2 p_{2}(t) Y_{2}^{+}(t) Z_{2}(t) d W(t) \\
\leq & -\alpha_{2}(t) p_{2}(t)\left[Y_{2}^{+}(t)\right]^{2}+2 p_{2}(t) Y_{2}^{+}(t) \mathbb{1}_{\left[Y_{2}(t)>0\right]}\left[f(t, y(t), z(t))-\widehat{f}_{2}(t, y(t), z(t))\right] \\
& \left.-\mathbb{1}_{\left[Y_{2}(t)>0\right]} p_{2}(t) Z_{2}^{2}(t)\right] d t-\mathbb{1}_{\left[Y_{2}(t)>0\right]} 2 p_{2}(t) Y_{2}^{+}(t) Z_{2}(t) d W(t)+2 p_{2}(t) c_{1}(t)\left[Y_{2}^{+}(t)\right]^{2} d t \\
& +\bar{\beta}_{2} c_{2}^{2}(t) p_{2}(t)\left[Y_{2}^{+}(t)\right]^{2} d t+\bar{\beta}_{2}^{-1} \mathbb{1}_{\left[Y_{2}(t)>0\right]} p_{2}(t) Z_{2}^{2}(t) d t \\
\leq & -2 \mathbb{1}_{\left[Y_{2}(t)>0\right]} p_{1}(t) Y_{2}^{+}(t) Z_{2}(t) d W(t), \\
&
\end{aligned}
$$

which in integral form becomes

$$
p_{2}(t)\left[Y_{2}^{+}(t)\right]^{2} \leq-\int_{t}^{T} 2 \mathbb{1}_{\left[Y_{2}(s)>0\right]} p_{2}(s) Y_{2}^{+}(s) Z_{2}(s) d W(s) .
$$

Since the stochastic integral on the right-hand side is a martingale due to Lemma 2.1 (ii), we have

$$
\mathbb{E}\left[p_{2}(t)\left[Y_{2}^{+}(t)\right]^{2}\right] \leq 0
$$

which concludes the proof. 


\section{Unbounded continuous generator}

In this section, we consider the one-dimensional version of equation (1) with a continuous $f$ with respect to $y$ and $z$, which satisfies a linear growth condition rather than the Lipschitz-type condition. The main idea here, as in [27], is to approximate the generator $f$ by an infinite sequence of Lipschitztype approximating functions. Each such a function generates a BSDE, and we show that the solutions to such a sequence of BSDEs converge to the solution of (1).

We say that the progressively measurable function $f$ and the random variable $\xi$, or the pair $(f, \xi)$, satisfies conditions $A 3$ if:

(i)' $d=1$ and $f(t, y, z)$ is a continuous function of $y$ and $z$;

(ii)' $|f(t, y, z)| \leq c_{0}(t)+c_{1}(t)|y|+c_{2}(t)|z|$, for all $y \in \mathbb{R}, z \in \mathbb{R}^{k},(t, \omega)$ a.e.;

(iii)' $\xi \in \widetilde{M}^{2}\left(\Omega, \mathscr{F}_{T}, \mathbb{P} ; \mathbb{R}\right)$;

(iv)' $c_{0}(\cdot) \in \widetilde{M}^{2}(0, T ; \mathbb{R})$ and $\left[c_{0}(\cdot) \widetilde{\alpha}(\cdot)^{-\frac{1}{2}}\right] \in \widetilde{M}^{2}(0, T ; \mathbb{R})$.

As already mentioned in the introduction, our assumption (ii)' permits for random and possibly unbounded coefficients $c_{1}(\cdot)$ and $c_{2}(\cdot)$, which is not the case in [39] and [40]. The results of the previous section are our main tools in dealing with the problem of solvability. We focus on utilizing Theorem 2.1 (i) and Theorem 2.2 (i) only, as similar results can be obtained by applying Theorem 2.1 (ii) and Theorem 2.2 (ii).

We introduce the sequence of functions

$f_{n}(t, y, z) \equiv \sup _{(u, v) \in \mathbb{R}^{1+k}}\left\{f(t, u, v)-\left[c_{1}(t)+n\right]|u-y|-\left[c_{2}(t)+n\right]|v-z|\right\}, \quad n \geq 1$,

which are clearly well-defined. Their main properties are summarized in the following result.

Lemma 3.1. (i) Linear growth: for any $y \in \mathbb{R}, z \in \mathbb{R}^{k},\left|f_{n}(t, y, z)\right| \leq$ $c_{0}(t)+c_{1}(t)|y|+c_{2}(t)|z| ;$

(ii) Monotonicity: $f_{n}$ is a decreasing function of $n$; 
(iii) Lipschitz condition: for any $y_{1}, y_{2} \in \mathbb{R}, z_{1}, z_{2} \in \mathbb{R}^{k}$,

$$
\left|f_{n}\left(t, y_{1}, z_{1}\right)-f_{n}\left(t, y_{2}, z_{2}\right)\right| \leq\left[c_{1}(t)+n\right]\left|y_{1}-y_{2}\right|+\left[c_{2}(t)+n\right]\left|z_{1}-z_{2}\right| ;
$$

(iv) Convergence: for any $y \in \mathbb{R}, z \in \mathbb{R}^{k}, \lim _{n \rightarrow \infty} f_{n}(t, y, z)=f(t, y, z)$.

Proof. (i) By the linear growth of $f$, for all $y \in \mathbb{R}, z \in \mathbb{R}^{k}$, we have

$$
\begin{aligned}
& f_{n}(t, y, z) \leq \sup _{(u, v) \in \mathbb{R}^{1+k}}\left\{|f(t, u, v)|-\left[c_{1}(t)+n\right]|u-y|-\left[c_{2}(t)+n\right]|v-z|\right\} \\
\leq & c_{0}(t)+\sup _{(u, v) \in \mathbb{R}^{1+k}}\left\{c_{1}(t)|u|+c_{2}(t)|v|-\left[c_{1}(t)+n\right]|u-y|-\left[c_{2}(t)+n\right]|v-z|\right\} \\
\leq & c_{0}(t)+\sup _{(u, v) \in \mathbb{R}^{1+k}}\left\{c_{1}(t)|u|+c_{2}(t)|v|-c_{1}(t)(|u|-|y|)-c_{2}(t)(|v|-|z|)\right\} \\
\leq & c_{1}(t)+c_{2}(t)|y|+c_{3}(t)|z| .
\end{aligned}
$$

The inequality $f_{n}(t, y, z) \geq-c_{0}(t)-c_{1}(t)|y|-c_{2}(t)|z|$ can be proved similarly.

(ii) This follows from the definition of $f_{n}$ itself.

(iii) By inequality $\left|\sup _{i \in I} a_{i}-\sup _{i \in I} b_{i}\right| \leq \sup _{i \in I}\left|a_{i}-b_{i}\right|$, with $I$ being an 
arbitrary index set, we have

$$
\begin{aligned}
& \left|f_{n}\left(t, y_{1}, z_{1}\right)-f_{n}\left(t, y_{2}, z_{2}\right)\right| \\
= & \mid \sup _{(u, v) \in \mathbb{R}^{1+k}}\left\{f(t, u, v)-\left[c_{1}(t)+n\right]\left|u-y_{1}\right|-\left[c_{2}(t)+n\right]\left|v-z_{1}\right|\right\} \\
& -\sup _{(u, v) \in \mathbb{R}^{1+k}}\left\{f(t, u, v)-\left[c_{1}(t)+n\right]\left|u-y_{2}\right|-\left[c_{2}(t)+n\right]\left|v-z_{2}\right|\right\} \mid \\
\leq & \sup _{(u, v) \in \mathbb{R}^{1+k}}\left|\left\{\left[c_{1}(t)+n\right]\left(\left|u-y_{2}\right|-\left|u-y_{1}\right|\right)+\left[c_{2}(t)+n\right]\left(\left|v-z_{2}\right|-\left|v-z_{1}\right|\right)\right\}\right| \\
\leq & \sup _{(u, v) \in \mathbb{R}^{1+k}}\left|\left\{\left[c_{1}(t)+n\right]\left|u-y_{2}-u+y_{1}\right|+\left[c_{2}(t)+n\right]\left|v-z_{2}-v+z_{1}\right|\right\}\right| \\
= & {\left[c_{1}(t)+n\right]\left|y_{1}-y_{2}\right|+\left[c_{2}(t)+n\right]\left|z_{1}-z_{2}\right| . }
\end{aligned}
$$$$
\text { (iv) For any } n \geq 1 \text {, there exists }\left(u_{n}, v_{n}\right) \in \mathbb{R}^{1+k} \text { such that }
$$$$
f_{n}(t, y, z) \leq f\left(t, u_{n}, v_{n}\right)-\left[c_{1}(t)+n\right]\left|u_{n}-y\right|-\left[c_{2}(t)+n\right]\left|v_{n}-z\right|+n^{-1} .
$$

In other words,

$$
f_{n}(t, y, z)+\left[c_{1}(t)+n\right]\left|u_{n}-y\right|+\left[c_{2}(t)+n\right]\left|v_{n}-z\right| \leq f\left(t, u_{n}, v_{n}\right)+n^{-1} .
$$

Note that in order to make the left-hand side of above inequality finite as $n \rightarrow \infty$, it is necessary to have $\lim _{n \rightarrow \infty}\left(u_{n}, v_{n}\right)=(y, z)$. And then

$$
\lim _{n \rightarrow \infty} f_{n}(t, y, z) \leq f(t, y, z)
$$

On the other hand, by the definition of $f_{n}$, we have $f_{n}(t, y, z) \geq f(t, y, z)$. Hence

$$
\lim _{n \rightarrow \infty} f_{n}(t, y, z)=f(t, y, z)
$$


Using functions $\left\{f_{n}\right\}_{n \geq 1}$ as generators, we introduce the following sequence of equations

$$
\widetilde{y}_{n}(t)=\xi+\int_{t}^{T} f_{n}\left(s, \widetilde{y}_{n}(s), \widetilde{z}_{n}(s)\right) d s-\int_{t}^{T} \widetilde{z}_{n}(s) d W(s), \quad t \in[0, T] .
$$

Lemma 3.2. If conditions A3 hold, then equations (21) have unique solution pairs $\left(\widetilde{y}_{n}(\cdot), \widetilde{z}_{n}(\cdot)\right) \in \widetilde{H}^{2}(0, T ; \mathbb{R}) \times \widetilde{M}^{2}\left(0, T ; \mathbb{R}^{k}\right)$, for any $n \geq 1$.

Proof. We only need to show that the assumptions of the previous section, which ensure the applicability of Theorem 2.1 (i), hold. Thus,

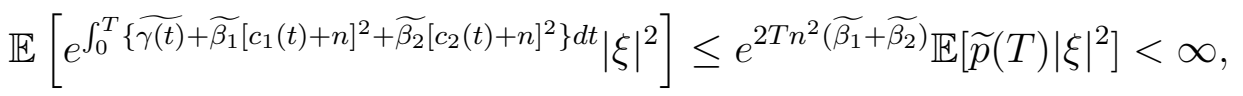

and

$$
\begin{aligned}
& \mathbb{E} \int_{0}^{T} e^{\int_{0}^{t}\left\{\widetilde{\gamma(t)}+\widetilde{\beta_{1}}\left[c_{1}(t)+n\right]^{2}+\widetilde{\beta_{2}}\left[c_{2}(t)+n\right]^{2}\right\} d s} \frac{\left|f_{n}(t, 0,0)\right|^{2}}{\widetilde{\gamma(t)}+\widetilde{\beta}_{1}\left[c_{1}(t)+n\right]^{2}+\widetilde{\beta}_{2}\left[c_{2}(t)+n\right]^{2}} d t \\
& \leq \mathbb{E} \int_{0}^{T} e^{2 n^{2} t\left(\widetilde{\beta_{1}}+\widetilde{\beta_{2}}\right)} \widetilde{p}(t) \frac{\left|c_{0}(t)\right|^{2}}{\widetilde{\gamma(t)}+\widetilde{\beta}_{1}\left[c_{1}(t)+n\right]^{2}+\widetilde{\beta_{2}}\left[c_{2}(t)+n\right]^{2}} d t \\
& \leq 2 e^{2 n^{2} T\left(\widetilde{\beta_{1}}+\widetilde{\beta_{2}}\right)} \mathbb{E} \int_{0}^{T} \widetilde{p}(t) \frac{\left|c_{0}(t)\right|^{2}}{\widetilde{\alpha}(t)} d t<\infty .
\end{aligned}
$$

Our main task now is to prove that the sequence of solutions $\left\{\widetilde{y}_{n}(\cdot), \widetilde{z}_{n}(\cdot)\right\}_{n \geq 1}$, converges to the solution $(y(\cdot), z(\cdot))$ of (1). We first present two useful lemmas.

Lemma 3.3. Let the conditions A3 hold. There exists a constant $\kappa$, independent of $n$, such that $\left\|\widetilde{\widetilde{y}_{n}}\right\| \leq \kappa$ and $\left\|\widetilde{\widetilde{z}_{n}}\right\| \leq \kappa$, for all $n \geq 1$.

Proof. From the fact that the sequence $f_{n}$ is decreasing, and Theorem 2.2 (i), we know that $\widetilde{y}_{1}(t) \geq \widetilde{y}_{2}(t) \geq \ldots, \forall t \in[0, T]$ a.s.. Hence, there exists a constant $\kappa_{1}$ such that

$$
\kappa_{1} \geq \widetilde{\widetilde{y}_{1} \|} \geq \widetilde{\widetilde{y}_{2} \|} \geq \ldots
$$


By making use the linear growth property of $f_{n}$, we obtain

$$
\begin{aligned}
& -d \widetilde{p}(t)\left|\widetilde{y}_{n}(t)\right|^{2} \\
& =-\widetilde{\alpha}(t) \widetilde{p}(t)\left|\widetilde{y}_{n}(t)\right|^{2} d t-\widetilde{p}(t)\left|\widetilde{z}_{n}(t)\right|^{2} d t-2 \widetilde{p}(t) \widetilde{y}_{n}(t) \widetilde{z}_{n}(t) d W(t) \\
& +2 \widetilde{p}(t) \widetilde{y}_{n}(t) f_{n}\left(t, \widetilde{y}_{n}(t), \widetilde{z}_{n}(t)\right) d t \\
& \leq-\widetilde{\alpha}(t) \widetilde{p}(t)\left|\widetilde{y}_{n}(t)\right|^{2} d t-\widetilde{p}(t)\left|\widetilde{z}_{n}(t)\right|^{2} d t-2 \widetilde{p}(t) \widetilde{y}_{n}(t) \widetilde{z}_{n}(t) d W(t) \\
& +2 \widetilde{p}(t)\left|\widetilde{y}_{n}(t)\right|\left|f_{n}\left(t, \widetilde{y}_{n}(t), \widetilde{z}_{n}(t)\right)\right| d t \\
& \leq-\widetilde{\alpha}(t) \widetilde{p}(t)\left|\widetilde{y}_{n}(t)\right|^{2} d t-\widetilde{p}(t)\left|\widetilde{z}_{n}(t)\right|^{2} d t-2 \widetilde{p}(t) \widetilde{y}_{n}(t) \widetilde{z}_{n}(t) d W(t) \\
& +2 \widetilde{p}(t)\left|\widetilde{y}_{n}(t)\right|\left[c_{0}(t)+c_{1}(t)\left|\widetilde{y}_{n}(t)\right|+c_{2}(t)\left|\widetilde{z}_{n}(t)\right|\right] d t \\
& \leq-\widetilde{\alpha}(t) \widetilde{p}(t)\left|\widetilde{y}_{n}(t)\right|^{2} d t-\widetilde{p}(t)\left|\widetilde{z}_{n}(t)\right|^{2} d t-2 \widetilde{p}(t) \widetilde{y}_{n}(t) \widetilde{z}_{n}(t) d W(t) \\
& +\widetilde{p}(t)\left|\widetilde{y}_{n}(t)\right|^{2} d t+\widetilde{p}(t) c_{0}^{2}(t) d t+\widetilde{\beta}_{1} c_{1}^{2}(t) \widetilde{p}(t)\left|\widetilde{y}_{n}(t)\right|^{2} d t+\left(\widetilde{\beta}_{1}\right)^{-1} \widetilde{p}(t)\left|\widetilde{y}_{n}(t)\right|^{2} d t \\
& +\widetilde{\beta}_{2} c_{2}^{2}(t) \widetilde{p}(t)\left|\widetilde{y}_{n}(t)\right|^{2}+\left(\widetilde{\beta}_{2}\right)^{-1} \widetilde{p}(t)\left|\widetilde{z}_{n}(t)\right|^{2} d t \\
& \leq-\left[1-\left(\widetilde{\beta}_{2}\right)^{-1}\right] \widetilde{p}(t)\left|\widetilde{z}_{n}(t)\right|^{2} d t+\widetilde{p}(t) c_{0}^{2}(t) d t \\
& +\left[1+\left(\widetilde{\beta}_{1}\right)^{-1}\right] \widetilde{p}(t)\left|\widetilde{y}_{n}(t)\right|^{2} d t-2 \widetilde{p}(t) \widetilde{y}_{n}(t) \widetilde{z}_{n}(t) d W(t),
\end{aligned}
$$


which in integral form becomes

$$
\begin{aligned}
\int_{t}^{T} \widetilde{p}(s)\left|\widetilde{z}_{n}(s)\right|^{2} d s & \leq \frac{\widetilde{p}(T) \xi^{2}}{\left[1-\left(\widetilde{\beta}_{2}\right)^{-1}\right]}+\frac{\int_{t}^{T} \widetilde{p}(s) c_{0}^{2}(s) d s}{\left[1-\left(\widetilde{\beta}_{2}\right)^{-1}\right]} \\
& +\frac{\left[1+\left(\widetilde{\beta}_{1}\right)^{-1}\right]}{\left[1-\left(\widetilde{\beta}_{2}\right)^{-1}\right]} \int_{t}^{T} \widetilde{p}(s)\left|\widetilde{y}_{n}(t)\right|^{2} d s-\frac{2 \int_{t}^{T} \widetilde{p}(s) \widetilde{y}_{n}(s) \widetilde{z}_{n}(s) d W(t)}{\left[1-\left(\widetilde{\beta}_{2}\right)^{-1}\right]} .
\end{aligned}
$$

From Lemma 2.1, we know that the stochastic integral on the right-hand side is a martingale. Taking the expectation of both sides gives

$$
\begin{aligned}
\widetilde{\widetilde{z}_{n} \|} & \leq \frac{\mathbb{E}\left[\widetilde{p}(T) \xi^{2}\right]}{\left[1-\left(\widetilde{\beta_{2}}\right)^{-1}\right]}+\frac{\mathbb{E} \int_{0}^{T} \widetilde{p}(s) c_{0}^{2}(s) d s}{\left[1-\left(\widetilde{\beta}_{2}\right)^{-1}\right]}+\frac{\left[1+\left(\widetilde{\beta}_{1}\right)^{-1}\right]}{\left[1-\left(\widetilde{\beta}_{2}\right)^{-1}\right]}\left\|\widetilde{\widetilde{y}_{n}}\right\| \\
& \leq \frac{\mathbb{E}\left[\widetilde{p}(T) \xi^{2}\right]}{\left[1-\left(\widetilde{\beta_{2}}\right)^{-1}\right]}+\frac{\mathbb{E} \int_{0}^{T} \widetilde{p}(s) c_{0}^{2}(s) d s}{\left[1-\left(\widetilde{\beta}_{2}\right)^{-1}\right]}+\frac{\left[1+\left(\widetilde{\beta_{1}}\right)^{-1}\right]}{\left[1-\left(\widetilde{\beta}_{2}\right)^{-1}\right]} \kappa_{1}=\kappa_{2} .
\end{aligned}
$$

Finally, $\kappa=\max \left(\kappa_{1}, \kappa_{2}\right)$.

Lemma 3.4. Let the conditions A3 hold. The pair of processes $\left(\widetilde{y}_{n}(\cdot), \widetilde{z}_{n}(\cdot)\right)_{n \geq 1}$ converges to $(\widetilde{y}(\cdot), \widetilde{z}(\cdot))$ in $\widetilde{M}^{2}(0, T ; \mathbb{R}) \times \widetilde{M}^{2}\left(0, T ; \mathbb{R}^{k}\right)$.

Proof. Let us consider a measurable and Lipchitz function $g(t, y, z)=-\left[c_{0}(t)+\right.$ $\left.c_{1}(t)|y|+c_{2}(t)|z|\right]$. From Lemma 3.1 (iii) and Theorem[2.1(i) we know the following BSDEs have a unique adapted solution on $\widetilde{H}^{2}(0, T ; \mathbb{R}) \times \widetilde{M}^{2}\left(0, T ; \mathbb{R}^{k}\right)$ :

$$
\widetilde{y}_{n}(t)=\xi+\int_{t}^{T} f_{n}\left(s, \widetilde{y}_{n}(s), \widetilde{z}_{n}(s)\right) d s-\int_{t}^{T} \widetilde{z}_{n}(s) d W(s), \quad t \in[0, T],
$$

and

$$
K(t)=\xi+\int_{t}^{T} g(s, K(s), L(s)) d s-\int_{t}^{T} L(s) d W(s), \quad t \in[0, T] .
$$

By Comparison Theorem 2.2 (i), we have

$$
K(t) \leq \widetilde{y}_{n}(t) \leq \widetilde{y}_{n-1}(t) \leq \widetilde{y}_{1}(t), \forall n \geq 1
$$


Hence $\left\{\widetilde{y}_{n}(t)\right\}_{n \geq 1}$ is decreasing and bounded in $\widetilde{M}^{2}(0, T ; \mathbb{R})$. Then by dominated convergence theorem, we know that $\left\{\widetilde{y}_{n}(t)\right\}_{n \geq 1}$ converges pointwisely to $\widetilde{y}(t)^{*}$ in $\widetilde{M^{2}}(0, T ; \mathbb{R})$.

Applying the Itô's formula to $p(t)\left|\widetilde{y}_{n}(t)-\widetilde{y}_{m}(t)\right|^{2}$ and writing in integral form, we have

$$
\begin{aligned}
& \widetilde{p}(t)\left|\widetilde{y}_{n}(t)-\widetilde{y}_{m}(t)\right|^{2}+\int_{t}^{T} \widetilde{p}(s)\left|\widetilde{z}_{n}(s)-\widetilde{z}_{m}(s)\right|^{2} d s \\
= & \int_{t}^{T} 2 \widetilde{p}(s)\left(\widetilde{y}_{n}(s)-\widetilde{y}_{m}(s)\right)\left[f_{n}\left(s, \widetilde{y}_{n}(s), \widetilde{z}_{n}(s)\right)-f_{m}\left(s, \widetilde{y}_{m}(s), \widetilde{z}_{m}(s)\right)\right] d s \\
+ & \int_{t}^{T}-2 \widetilde{p}(s)\left(\widetilde{y}_{n}(s)-\widetilde{y}_{m}(s)\right)\left(\widetilde{z}_{n}(s)-\widetilde{z}_{m}(s)\right) d W(s) .
\end{aligned}
$$

Taking expectations on both sides, from Lemma 2.1 (ii) and using the linear 
growth property of $f_{n}$ and $f_{m}$, we have

$$
\begin{aligned}
& \mathbb{E}\left[\int_{t}^{T} \widetilde{p}(s)\left|\widetilde{z}_{n}(s)-\widetilde{z}_{m}(s)\right|^{2} d s\right] \\
& \leq 2 \mathbb{E}\left[\int_{t}^{T} \widetilde{p}(s)\left(\widetilde{y}_{n}(s)-\widetilde{y}_{m}(s)\right)\left[f_{n}\left(s, \widetilde{y}_{n}(s), \widetilde{z}_{n}(s)\right)-f_{m}\left(s, \widetilde{y}_{m}(s), \widetilde{z}_{m}(s)\right)\right] d s\right] \\
& \leq 2 \mathbb{E}\left[\int_{t}^{T} \sqrt{\widetilde{\alpha}(s) \widetilde{p}(s)}\left(\widetilde{y}_{n}(s)-\widetilde{y}_{m}(s)\right) \frac{\sqrt{\widetilde{p}(s)}}{\sqrt{\widetilde{\alpha}(s)}}\left[f_{n}\left(s, \widetilde{y}_{n}(s), \widetilde{z}_{n}(s)\right)-f_{m}\left(s, \widetilde{y}_{m}(s), \widetilde{z}_{m}(s)\right)\right] d s\right] \\
& \leq 2\left(\mathbb{E}\left[\int_{t}^{T} \widetilde{\alpha}(s) \widetilde{p}(s)\left|\widetilde{y}_{n}(s)-\widetilde{y}_{m}(s)\right|^{2} d s\right]\right)^{\frac{1}{2}} \\
& \cdot\left(\mathbb{E}\left[\int_{t}^{T} \frac{\widetilde{p}(s)}{\widetilde{\alpha}(s)}\left|f_{n}\left(s, \widetilde{y}_{n}(s), \widetilde{z}_{n}(s)\right)-f_{m}\left(s, \widetilde{y}_{m}(s), \widetilde{z}_{m}(s)\right)\right|^{2} d s\right]\right)^{\frac{1}{2}} \\
& \leq 2 \sqrt{2}\left(\mathbb{E}\left[\int_{t}^{T} \widetilde{\alpha}(s) \widetilde{p}(s)\left|\widetilde{y}_{n}(s)-\widetilde{y}_{m}(s)\right|^{2} d s\right]\right)^{\frac{1}{2}}\left(\mathbb { E } \left[\int _ { t } ^ { T } \frac { \widetilde { p } ( s ) } { \widetilde { \alpha } ( s ) } \left[\left|c_{0}(s)+c_{1}(s)\right| \widetilde{y}_{n}(s) \mid\right.\right.\right. \\
& \left.\left.\left.+\left.c_{2}(s)\left|\widetilde{z}_{n}(s)\right|\right|^{2}+\left|c_{0}(s)+c_{1}(s)\right| \widetilde{y}_{m}(s)\left|+c_{2}(s)\right| \widetilde{z}_{m}(s)||^{2}\right] d s\right]\right)^{\frac{1}{2}} \\
& \leq 2 \sqrt{6}\left(\mathbb{E}\left[\int_{t}^{T} \widetilde{\alpha}(s) \widetilde{p}(s)\left|\widetilde{y}_{n}(s)-\widetilde{y}_{m}(s)\right|^{2} d s\right]\right)^{\frac{1}{2}}\left(\mathbb { E } \left[\int _ { t } ^ { T } \frac { \widetilde { p } ( s ) } { \widetilde { \alpha } ( s ) } \left[2 c_{0}^{2}(s)+c_{1}^{2}(s)\left|\widetilde{y}_{n}(s)\right|^{2}\right.\right.\right. \\
& \left.\left.\left.+c_{2}^{2}(s)\left|\widetilde{z}_{n}(s)\right|^{2}+c_{1}^{2}(s)\left|\widetilde{y}_{m}(s)\right|^{2}+c_{2}^{2}(s)\left|\widetilde{z}_{m}(s)\right|^{2}\right] d s\right]\right)^{\frac{1}{2}}
\end{aligned}
$$




$$
\begin{aligned}
\leq & 2 \sqrt{6}\left(\mathbb{E}\left[\int_{t}^{T} \widetilde{\alpha}(s) \widetilde{p}(s)\left|\widetilde{y}_{n}(s)-\widetilde{y}_{m}(s)\right|^{2} d s\right]\right)^{\frac{1}{2}}\left(\mathbb { E } \left[\int _ { t } ^ { T } \widetilde { p } ( s ) \left[\frac{2 c_{0}^{2}(s)}{\widetilde{\alpha}(s)}+\left|\widetilde{y}_{n}(s)\right|^{2}\right.\right.\right. \\
& \left.\left.\left.+\left|\widetilde{z}_{n}(s)\right|^{2}+\left|\widetilde{y}_{m}(s)\right|^{2}+\left|\widetilde{z}_{m}(s)\right|^{2}\right] d s\right]\right)^{\frac{1}{2}} \\
\leq & 2 \sqrt{6} \widetilde{\kappa}\left(\mathbb{E}\left[\int_{t}^{T} \widetilde{\alpha}(s) \widetilde{p}(s)\left|\widetilde{y}_{n}(s)-\widetilde{y}_{m}(s)\right|^{2} d s\right]\right)^{\frac{1}{2}},
\end{aligned}
$$

where $\widetilde{\kappa} \equiv\left(4 \kappa+\left\|\widetilde{c_{0}(\cdot) \alpha(\cdot)^{-\frac{1}{2}}}\right\|\right)^{\frac{1}{2}}$. Therefore, this, together with the fact that $\left\{\widetilde{y}_{n}(t)\right\}_{n \geq 1}$ pointwisely converges in $\widetilde{M}^{2}(0, T ; \mathbb{R})$, implies that $\left\{\widetilde{z}_{n}(t)\right\}_{n \geq 1}$ is a Cauchy sequence in $\widetilde{M^{2}}\left(0, T ; \mathbb{R}^{k}\right)$ and then converges to $\widetilde{z}(t)^{*}$ in the same space.

Now we present the main result in this section.

Theorem 3.1. (Existence) Equation (1) has an adapted solution $(\widetilde{y}(\cdot), \widetilde{z}(\cdot)) \in$ $\widetilde{M}^{2}(0, T ; \mathbb{R}) \times \widetilde{M}^{2}\left(0, T ; \mathbb{R}^{k}\right)$, which is also a maximal solution, i.e. for any other solution $(\bar{y}(\cdot), \bar{z}(\cdot))$ of equation (1), we have $\widetilde{y}(\cdot) \geq \bar{y}(\cdot)$.

Proof. Similar to previous calculation in Lemma 3.4, taking supremum over $t$ for equation (23) and using the Burkholder-Davis-Gundy's inequality, we 
have

$$
\begin{aligned}
& \mathbb{E}\left[\sup _{t \in[0, T]} \widetilde{p}(t)\left|\widetilde{y}_{n}(t)-\widetilde{y}_{m}(t)\right|^{2}\right] \\
& \leq \mathbb{E}\left[\sup _{t \in[0, T]} \int_{t}^{T} 2 \widetilde{p}(s)\left(\widetilde{y}_{n}(s)-\widetilde{y}_{m}(s)\right)\left[f_{n}\left(s, \widetilde{y}_{n}(s), \widetilde{z}_{n}(s)\right)-f_{m}\left(s, \widetilde{y}_{m}(s), \widetilde{z}_{m}(s)\right)\right] d s\right] \\
& +\mathbb{E}\left[\sup _{t \in[0, T]} \int_{t}^{T}-2 \widetilde{p}(s)\left(\widetilde{y}_{n}(s)-\widetilde{y}_{m}(s)\right)\left(\widetilde{z}_{n}(s)-\widetilde{z}_{m}(s)\right) d W(s)\right] \\
& \leq 2 \mathbb{E}\left[\int_{0}^{T} \widetilde{p}(s)\left|\widetilde{y}_{n}(s)-\widetilde{y}_{m}(s)\right|\left|f_{n}\left(s, \widetilde{y}_{n}(s), \widetilde{z}_{n}(s)\right)-f_{m}\left(s, \widetilde{y}_{m}(s), \widetilde{z}_{m}(s)\right)\right| d s\right] \\
& +K \mathbb{E}\left[\int_{0}^{T}\left|\sqrt{\widetilde{p}(s)}\left(\widetilde{y}_{n}(s)-\widetilde{y}_{m}(s)\right)\right|^{2}\left|\sqrt{\widetilde{p}(s)}\left(\widetilde{z}_{n}(s)-\widetilde{z}_{m}(s)\right)\right|^{2} d s\right]^{\frac{1}{2}} \\
& \leq 2\left(\mathbb{E}\left[\int_{t}^{T} \widetilde{\alpha}(s) \widetilde{p}(s)\left|\widetilde{y}_{n}(s)-\widetilde{y}_{m}(s)\right|^{2} d s\right]\right)^{\frac{1}{2}} \\
& \cdot\left(\mathbb{E}\left[\int_{t}^{T} \frac{\widetilde{p}(s)}{\widetilde{\alpha}(s)}\left|f_{n}\left(s, \widetilde{y}_{n}(s), \widetilde{z}_{n}(s)\right)-f_{m}\left(s, \widetilde{y}_{m}(s), \widetilde{z}_{m}(s)\right)\right|^{2} d s\right]\right)^{\frac{1}{2}} \\
& +\frac{K}{2} \mathbb{E}\left[\sup _{t \in[0, T]}\left|\sqrt{\widetilde{p}(s)}\left(\widetilde{y}_{n}(s)-\widetilde{y}_{m}(s)\right)\right|^{2}+\int_{t}^{T}\left|\sqrt{\widetilde{p}(s)}\left(\widetilde{z}_{n}(s)-\widetilde{z}_{m}(s)\right)\right|^{2} d s\right] \\
& \leq 2 \sqrt{6} \widetilde{\kappa}\left(\mathbb{E}\left[\int_{t}^{T} \widetilde{\alpha}(s) \widetilde{p}(s)\left|\widetilde{y}_{n}(s)-\widetilde{y}_{m}(s)\right|^{2} d s\right]\right)^{\frac{1}{2}} \\
& +\frac{K}{2} \mathbb{E}\left[\sup _{t \in[0, T]} \widetilde{p}(s)\left|\widetilde{y}_{n}(s)-\widetilde{y}_{m}(s)\right|^{2}+\int_{t}^{T} \widetilde{p}(s)\left|\widetilde{z}_{n}(s)-\widetilde{z}_{m}(s)\right|^{2} d s\right] .
\end{aligned}
$$


Therefore by Lemma 3.4 , for any $t \in[0, T],\left\{\widetilde{y}_{n}(t)\right\}_{n \geq 1}$ converges uniformly in $\widetilde{M}^{2}(0, T ; \mathbb{R})$ to $y(t)$ in the same space. Since $\left\{\widetilde{y}_{n}(t)\right\}_{n \geq 1}$ is continuous, by the uniform convergence theorem, $\widetilde{y}(t)$ is a continuous process.

Now we show that the sequence of processes $\left\{f_{n}\left(t, \widetilde{y}_{n}(t), \widetilde{z}_{n}(t)\right)\right\}_{n \geq 1}$ converges to $\{f(t, \widetilde{y}(t), \widetilde{z}(t))\}$ in $L_{\mathscr{F}}(0, T ; \mathbb{R})$. Note that for any positive and large enough $\delta$,

$$
\begin{aligned}
& \mathbb{E}\left[\int_{0}^{T}\left|f_{n}\left(s, \widetilde{y}_{n}(s), \widetilde{z}_{n}(s)\right)-f(s, \widetilde{y}(s), \widetilde{z}(s))\right| d s\right] \\
= & \mathbb{E}\left[\int_{0}^{T}\left|f_{n}\left(s, \widetilde{y}_{n}(s), \widetilde{z}_{n}(s)\right)-f(s, \widetilde{y}(s), \widetilde{z}(s))\right| \mathbb{1}_{\left\{\frac{c_{1}(s)\left|\widetilde{y}_{n}(s)\right|+c_{2}(s)\left|\widetilde{z}_{n}(s)\right|}{c_{0}^{2}(s)+c_{1}^{2}(s)+c_{2}^{2}(s)} \leq \delta\right\}} d s\right] \\
+ & \left.\mathbb{E}\left[\int_{0}^{T}\left|f_{n}\left(s, \widetilde{y}_{n}(s), \widetilde{z}_{n}(s)\right)-f(s, \widetilde{y}(s), \widetilde{z}(s))\right| \mathbb{1}_{\left\{\frac{c_{1}(s)\left|\tilde{y}_{n}(s)\right|+c_{2}(s)\left|\tilde{z}_{n}(s)\right|}{c_{0}^{2}(s)+c_{1}^{2}(s)+c_{2}^{2}(s)}>\delta\right\}}\right\}\right] \\
\leq & \mathbb{E}\left[\int_{0}^{T}\left|f_{n}\left(s, \widetilde{y}_{n}(s), \widetilde{z}_{n}(s)\right)-f\left(s, \widetilde{y}_{n}(s), \widetilde{z}_{n}(s)\right)\right| \mathbb{1}_{\left\{\frac{c_{1}(s)\left|\widetilde{y}_{n}(s)\right|+c_{2}(s)\left|\tilde{z}_{n}(s)\right|}{c_{0}^{2}(s)+c_{1}^{2}(s)+c_{2}^{2}(s)} \leq \delta\right\}} d s\right] \\
+ & \mathbb{E}\left[\int_{0}^{T}\left|f\left(s, \widetilde{y}_{n}(s), \widetilde{z}_{n}(s)\right)-f(s, \widetilde{y}(s), \widetilde{z}(s))\right| \mathbb{1}_{\left\{\frac{c_{1}(s)\left|\widetilde{y}_{n}(s)\right|+c_{2}(s)\left|\tilde{z}_{n}(s)\right|}{c_{0}^{2}(s)+c_{1}^{2}(s)+c_{2}^{2}(s)} \leq \delta\right\}} d s\right] \\
+ & {\left[\int_{0}^{T}\left|f_{n}\left(s, \widetilde{y}_{n}(s), \widetilde{z}_{n}(s)\right)-f(s, \widetilde{y}(s), \widetilde{z}(s))\right| \mathbb{1}_{\left\{\frac{c_{1}(s)\left|\widetilde{y}_{n}(s)\right|+c_{2}(s)\left|\tilde{z}_{n}(s)\right|}{c_{0}^{2}(s)+c_{1}^{2}(s)+c_{2}^{2}(s)}>\delta\right\}} d s\right] . }
\end{aligned}
$$

By (ii), (iii) and (iv) in Lemma 3.1, assumption (i)' and the Dini's Theorem, as $n \rightarrow \infty$, we have

$$
\sup _{\left\{\frac{c_{1}(s)\left|\widetilde{y}_{n}(s)\right|+c_{2}(s)\left|\tilde{z}_{n}(s)\right|}{c_{0}^{2}(s)+c_{1}^{2}(s)+c_{2}^{2}(s)} \leq \delta\right\}}\left|f_{n}(t, \widetilde{y}(t), \widetilde{z}(t))-f(t, \widetilde{y}(t), \widetilde{z}(t))\right| \longrightarrow 0 .
$$

Therefore by the dominated convergence theorem, the first term in right hand side uniformly converges to 0. Due to assumption (i)', at least along a subsequence, the second term in the right hand side converges to 0. For the final term in the right hand side, by Lemma 3.1 (iii), assumption (ii)' and Lemma 3.3, together with the fact that $a \mathbb{1}_{\{X>a\}}<X$ for any nonnegative 
random variable $X$ and $a>0$, we have

$$
\begin{aligned}
& \mathbb{E}\left[\int_{0}^{T}\left|f_{n}\left(t, \widetilde{y}_{n}(s), \widetilde{z}_{n}(s)\right)-f(t, \widetilde{y}(s), \widetilde{z}(s))\right| \mathbb{1}_{\left\{\frac{c_{1}(s)\left|\tilde{y}_{n}(s)\right|+c_{2}(s)\left|\tilde{z}_{n}(s)\right|}{c_{0}^{2}(s)+c_{1}^{2}(s)+c_{2}^{2}(s)}>\delta\right\}} d s\right] \\
& \leq \mathbb{E}\left[\int_{0}^{T}\left[2 c_{0}(s)+c_{1}(s)\left|\widetilde{y}_{n}(s)\right|+c_{2}(s)\left|\widetilde{z}_{n}(s)\right|+c_{1}(s)|\widetilde{y}(s)|+c_{2}(s) \mid \widetilde{z}(s)\right] \mathbb{1}_{\left\{\frac{c_{1}(s)\left|\widetilde{y}_{n}(s)\right|+c_{2}(s)\left|\tilde{z}_{n}(s)\right|}{c_{0}^{2}(s)+c_{1}^{2}(s)+c_{2}^{2}(s)}>\delta\right\}} d s\right] \\
& \leq \mathbb{E}\left[\int_{0}^{T}\left[2 c_{0}(s)+c_{1}(s)\left|\widetilde{y}_{n}(s)\right|+c_{2}(s)\left|\widetilde{z}_{n}(s)\right|+c_{1}(s)|\widetilde{y}(s)|+c_{2}(s) \mid \widetilde{z}(s)\right]\right. \\
& \left.\frac{c_{1}(s)\left|\widetilde{y}_{n}(s)\right|+c_{2}(s)\left|\widetilde{z}_{n}(s)\right|}{\delta\left(c_{0}^{2}(s)+c_{1}^{2}(s)+c_{2}^{2}(s)\right)} d s\right] \\
& =\frac{1}{\delta} \mathbb{E}\left[\int _ { 0 } ^ { T } \frac { 1 } { c _ { 0 } ^ { 2 } ( s ) + c _ { 1 } ^ { 2 } ( s ) + c _ { 2 } ^ { 2 } ( s ) } \left[2 c_{0}(s) c_{1}(s)\left|\widetilde{y}_{n}(s)\right|+c_{1}^{2}(s)\left|\widetilde{y}_{n}(s)\right|^{2}+c_{1}(s) c_{2}(s)\left|\widetilde{y}_{n}(s)\right|\left|\widetilde{z}_{n}(s)\right|\right.\right. \\
& +c_{1}^{2}(s)\left|\widetilde{y}_{n}(s)\right||\widetilde{y}(s)|+c_{1}(s) c_{2}(s)\left|\widetilde{y}_{n}(s)\right||\widetilde{z}(s)|+2 c_{0}(s) c_{2}(s)\left|\widetilde{z}_{n}(s)\right|+c_{1}(s) c_{2}(s)\left|\widetilde{y}_{n}(s)\right|\left|\widetilde{z}_{n}(s)\right| \\
& \left.\left.+c_{2}^{2}(s)\left|\widetilde{z}_{n}(s)\right|^{2}+c_{1}(s) c_{2}(s)|\widetilde{y}(s)|\left|\widetilde{z}_{n}(s)\right|+c_{2}^{2}(s)\left|\widetilde{z}_{n}(s)\right||\widetilde{z}(s)|\right] d s\right] \\
& \leq \frac{1}{\delta} \mathbb{E}\left[\int _ { 0 } ^ { T } \frac { 1 } { c _ { 0 } ^ { 2 } ( s ) + c _ { 1 } ^ { 2 } ( s ) + c _ { 2 } ^ { 2 } ( s ) } \left[2 c_{0}^{2}(s)+4 c_{1}^{2}(s)\left|\widetilde{y}_{n}(s)\right|^{2}+4 c_{2}^{2}(s)\left|\widetilde{z}_{n}(s)\right|^{2}\right.\right. \\
& \left.\left.+c_{1}^{2}(s)|\widetilde{y}(s)|^{2}+c_{2}^{2}(s)|\widetilde{z}(s)|^{2}\right] d s\right] \\
& \leq \frac{1}{\delta} \mathbb{E}\left[\int_{0}^{T}\left[2+4\left|\widetilde{y}_{n}(s)\right|^{2}+4\left|\widetilde{z}_{n}(s)\right|^{2}+|\widetilde{y}(s)|^{2}+|\widetilde{z}(s)|^{2}\right] d s\right] \leq \frac{\bar{\kappa}}{\delta}
\end{aligned}
$$

where $\bar{\kappa}$ is a constant independent of $n$. Hence taking limits in the following 
equation

$$
\widetilde{y}_{n}(t)=\xi+\int_{t}^{T} f_{n}\left(s, \widetilde{y}_{n}(s), \widetilde{z}_{n}(s)\right) d s-\int_{t}^{T} \widetilde{z}_{n}(s) d W(s), \quad t \in[0, T] .
$$

we deduce that $(\widetilde{y}(t), \widetilde{z}(t)) \in \widetilde{M}^{2}(0, T ; \mathbb{R}) \times \widetilde{M}^{2}\left(0, T ; \mathbb{R}^{k}\right)$ is an adapted solution of equation (11).

Furthermore, suppose that $(\bar{y}(t), \bar{z}(t)) \in \widetilde{M}^{2}(0, T ; \mathbb{R}) \times \widetilde{M}^{2}\left(0, T ; \mathbb{R}^{k}\right)$ is any solution of equation (11). By the comparison theorem 2.2, we have $\widetilde{y}_{n}(t) \geq \bar{y}(t)$ for any $n \geq 1$ and then $\widetilde{y}(t) \geq \bar{y}(t)$. Hence $\widetilde{y}(t)$ is a maximal solution of equation (11).

\section{Conclusions}

We have considered two classes of BSDEs with possibly unbounded generator. The first class has a Lipschitz-type generator, whereas the second class has a continuous generator that satisfies a certain linear growth condition. In both cases we have given sufficient conditions for solvability. We expect that these results will prove to be useful in tackling more difficult problems with unbounded generator, such as the BSDEs with a quadratic growth and the Riccati BSDE, which play a fundamental role in stochastic control.

\section{Appendix}

Here we include the derivation of the lower bound for the parameter $\beta$ that appears in [16]. We do so for the readers' convenience, since in Theorem 6.1 of [16] no explicit lower bound is given, it is only assumed that parameter $\beta$ should be large enough. The notation of [16] will be used.

Equation (6.5) of [16] states that for some constants $k$ and $k^{\prime}$ the following holds

$$
\|(y, \eta)\|_{\beta}^{2}=k\|\xi\|_{\beta}^{2}+\frac{k^{\prime}}{\beta}\left\|\frac{f}{\alpha}\right\|_{\beta}^{2}
$$

where the definitions of these norms are given in [16], and are just weighted Euclidian norms. From equation (5.5) of [16], which gives the definition of 
the norm $\|(y, \eta)\|_{\beta}^{2}$, and the conclusions of Lemma 6.2 of [16], we obtain

$$
\begin{aligned}
\|(y, \eta)\|_{\beta}^{2} & =\|\alpha y\|_{\beta}^{2}+\|\eta\|_{\beta}^{2} \\
& \leq \frac{2}{\beta}\|\xi\|_{\beta}^{2}+\frac{8}{\beta^{2}}\left\|\frac{f}{\alpha}\right\|_{\beta}^{2}+18\|\xi\|_{\beta}^{2}+\frac{45}{\beta}\left\|\frac{f}{\alpha}\right\|_{\beta}^{2} \\
& =\left(18+\frac{2}{\beta}\right)\|\xi\|_{\beta}^{2}+\left(\frac{45}{\beta}+\frac{8}{\beta^{2}}\right)\left\|\frac{f}{\alpha}\right\|_{\beta}^{2} .
\end{aligned}
$$

Comparing (25) and (26) gives $k^{\prime}=45+\frac{8}{\beta}$.

Equation (6.16) of [16] states that for some constants $\tilde{k}$ and $\tilde{k}^{\prime}$ the following holds

$$
\|(\delta Y, \delta Z, \delta N)\|_{\beta}^{2} \leq \tilde{k}\|\delta \xi\|_{\beta}^{2}+\frac{\tilde{k}^{\prime}}{\beta}\left\|\frac{\delta_{2} f}{\alpha}\right\|_{\beta}^{2} .
$$

Here, different from [16], we have used the tilde notation for the constants $k$ and $k^{\prime}$ in order to avoid the clash of notation with these constants introduced in the previous paragraph. By inequality (6.5) of [16], we obtain that

$$
\begin{aligned}
\|(\delta Y, \delta Z, \delta N)\|_{\beta}^{2} & \leq k\|\delta \xi\|_{\beta}^{2}+\frac{k^{\prime}}{\beta}\left\|\frac{\varphi_{t}}{\alpha_{t}^{2}}\right\|_{\beta}^{2} \\
& \leq k\|\delta \xi\|_{\beta}^{2}+\frac{3 k^{\prime}}{\beta}\left(\|\alpha \delta Y\|_{\beta}^{2}+\left\|m^{*} \delta Z\right\|_{\beta}^{2}+\left\|\frac{\delta_{2} f}{\alpha}\right\|_{\beta}^{2}\right) \\
& \leq k\|\delta \xi\|_{\beta}^{2}+\frac{3 k^{\prime}}{\beta}\left(k\|\delta \xi\|_{\beta}^{2}+\frac{k^{\prime}}{\beta}\left\|\frac{\delta_{2} f}{\alpha}\right\|_{\beta}^{2}+\left\|\frac{\delta_{2} f}{\alpha}\right\|_{\beta}^{2}\right) \\
& =\left(k+\frac{3 k k^{\prime}}{\beta}\right)\|\delta \xi\|_{\beta}^{2}+\frac{3 k^{\prime}}{\beta}\left(\frac{k^{\prime}}{\beta}+1\right)\left\|\frac{\delta_{2} f}{\alpha}\right\|_{\beta}^{2} .
\end{aligned}
$$

Comparing (27) and (28) gives $\widetilde{k^{\prime}}=3 k^{\prime}\left(\frac{k^{\prime}}{\beta}+1\right)$.

The inequality at the end of page 35 of [16] is

$$
\|\alpha \delta Y\|_{\beta}^{2}+\left\|m^{*} \delta Z\right\|_{\beta}^{2} \leq \frac{\widehat{k^{\prime}}}{\beta}\|\alpha \delta y\|_{\beta}^{2}+\left\|m^{*} \delta z\right\|_{\beta}^{2},
$$


where we have used the hat notation for the constant $k^{\prime}$ in order to avoid the clash of notation with this constant introduced earlier. Similarly to the previous paragraph we obtain

$$
\begin{aligned}
\|(\delta Y, \delta Z)\|_{\beta}^{2} & =\|\alpha \delta Y\|_{\beta}^{2}+\left\|m^{*} \delta Z\right\|_{\beta}^{2} \leq \frac{\widetilde{k^{\prime}}}{\beta}\left\|\frac{\varphi}{\alpha}\right\|_{\beta}^{2} \\
& \leq \frac{3 \widetilde{k^{\prime}}}{\beta}\|\alpha \delta y\|_{\beta}^{2}+\left\|m^{*} \delta z\right\|_{\beta}^{2} .
\end{aligned}
$$

Comparing (29) and (30) gives $\widehat{k}^{\prime}=3 \widetilde{k}^{\prime}=9 k^{\prime}\left(\frac{k^{\prime}}{\beta}+1\right)$. In order to apply the contraction mapping principle, it is necessary to have $\frac{\widehat{k^{\prime}}}{\beta}<1$, i.e.

$$
9\left(\frac{45}{\beta}+\frac{8}{\beta^{2}}\right)\left(\frac{45}{\beta}+\frac{8}{\beta^{2}}+1\right)<1 .
$$

By solving above inequality for $\beta>0$, we obtain that by large enough in [16] it is meant that $\beta>446.05$.

\section{Acknowledgement}

We are grateful to the reviewer for a careful reading of the paper and suggestions that led to an improved version.

\section{References}

[1] K. Bahlali, Backward stochastic differential equations with locally Lipschitz coefficient, C. R. Acad. Sci. Paris, Ser. I 333 (2001), 481-486.

[2] K. Bahlali, S. Hamadène, and B. Mezerdi, Backward stochastic differential equations with two reflecting barriers and continuous with quadratic growth coefficient, Stochastic Processes and Their Applications, 115 (2005), 1107-1129.

[3] P. Barrieu and N. El Karoui, Monotone stability of quadratic semimartingales with applications to unbounded general quadratic BSDEs, Ann. Probab., 41 (2013), 1831-1863. 
[4] N. H. Bingham and R. Kiesel, Risk-neutral Valuation, Springer Finance, Second Edition, 2004.

[5] J.-M. Bismut, Linear Quadratic Optimal Stochastic Control with Random Coefficients, SIAM J. Control and Optimization, 14 (1976), 419444.

[6] P. Briand and Y. Hu, BSDE with quadratic growth and unbounded terminal value, Probab. Theory Related Fields, 136 (2006), 604-618.

[7] P. Briand and Y. Hu, Quadratic BSDEs with convex generators and unbounded terminal conditions, Probab. Theory Related Fields, 141 (2008), 543-567.

[8] P. Briand, J.-P. Lepeltier, and J. S. Martin, One-dimensional backward stochastic differential equations whose coefficient is monotonic in $y$ and non-Lipschitz in z, Bernoulli, 13 (2007), 80-91.

[9] P. Briand and A. Richou, On the uniqueness of solutions to quadratic BSDEs with non-convex generators, https://arxiv.org/pdf/1801.0015\%.pdf, 2017.

[10] R. L. Buckdahn, Backward stochastic differential equations driven by a martingale, 1993, preprint.

[11] P. Date and B. Gashi, Risk-sensitive control for a class of nonlinear stochastic systems with multilicative noise, Systems \& Control Letters, 62 (2013), 989-999.

[12] F. Delbaen and S. Tang, Harmonic analysis of stochastic systems and backward stochastic differential equations, Probab. Theory Relat. Fields, 146 (2010), 291-336.

[13] F. Delbaen, Y. Hu, and A. Richou, On the uniqueness of solutions to quadratic BSDEs with convex generators and unbounded terminal conditions, Annales de I'institut Henri Poincaré, (B) 47 (2011), 559-574.

[14] F. Delbaen, Y. Hu, and A. Richou, On the uniqueness of solutions to quadratic BSDEs with convex generators and unbounded terminal conditions: The critical case, Discrete and Continuous Dynamical SystemsSeries A, 35 (2015), 5273-5283. 
[15] S. J. Fan and L. Jiang, Existence and uniqueness result for a backward stochastic differential equation whose generator is Lipschitz continous in $y$ and uniformly continuous in z, J. Appl. Math. Comput. 36 (2011), $1-10$.

[16] N. El Karoui and S. Huang, A general result of existence and uniqueness of backward stochastic differential equations, In: El Karoui, N., Mazliak, A. (eds.) Backward Stochastic Differential Equations, Pitman research Notes in Math. Series, 364 (1997) 27-36.

[17] N. El Karoui, S. Peng, and M.C. Quenez, Backward stochastic differential equations in finance, Math. Finance, 7 (1997), 1-71.

[18] N. El Karoui, A. Matoussi, A. Ngoupeyou, Quadratic Exponential Semimartingales and Application to BSDE with jumps, arXiv:1603.0691, 2016.

[19] M. Koblyanski, Backward stochastic differential equations and partial differential equations with a quadraatic growth, Ann. Probab., 28 (2000), 558-602.

[20] B. Gashi, Stochastic minimum-energy control, Systems $\&$ Control Letters, 85 (2015), 70-76.

[21] B. Gashi and A. A. Pantelous, Linear backward stochastic differential equations of descriptor type: regular systems, Journal of Stochastic Analysis and Applications, 31 (2013), 142-166.

[22] B. Gashi, and A. A. Pantelous, Linear backward stochastic differential systems of descriptor type with structure and applications to engineering, Probabilistic Engineering Mechanics, 40 (2015), 1-11.

[23] S. Hamadène, J. P. Lepeltier, S. Peng, BSDEs with continuous coefficeints and application to Markovian nonzero sum stochastic differential games, in: N. El Karoui, L. Mazliak (Eds.), Pitman Research Notes in Mathematics Series, 364 (1997), 161-175.

[24] G. Jia, A uniqueness theorem for the solution of Backward Stochastic Differential Equations, C. R. Acad. Sci. Paris, Ser. I, 346 (2008) 439444. 
[25] G. Jia, Some uniqueness results for one-dimensional BSDEs with uniformly continuous coefficients, Statistics \& Probability Letters, 79 (2009), 436-441.

[26] F. C. Klebaner, Introduction to stochastic calculus with applications, Second edition (2005), Imerical College Press.

[27] J. P. Lepeltier and J. San Martin, Backward stochastic differential equations with continuous coefficient, Statistics 83 Probability Letters, 32 (1997), 425-430.

[28] J. P. Lepeltier and J. San Martin, Existence for BSDE with a superlinear quadratic coefficients, Stochastics and Stochastic Reports, 63 (1998), 227-240.

[29] J. Ma and J. Yong, Forward-Backward stochastic differential equations and their applications. Lecture Notes in Math. 1702, (1999) SpringerVerlag, New York.

[30] X. Mao, Adapted solution of backward stochastic differential equations with non-lipschitz coefficients, Stochastic Processes and Applications, 58, (1995), 281-292.

[31] X. Mao, Stochastic Differential Equations and Their Applications, Second Edition (2011), Woodhead Publishing.

[32] M. Mocha and N. Westray, Quadratic semimartingale BSDEs under an exponential moments condition, Séminaire de Probabilités XLIV, Lecture Notes in Math., Springer, 105-139, 2012.

[33] M.-A. Morlais, Quadratic BSDEs driven by a continuous martingale and applications to the utility maximization problem, Finance Soch., 13 (2009), 121-150.

[34] E. Pardoux and S. Peng, 1990. Adapted solution of a backward stochastic differential equation, Systems 83 Control Letters, 14 (1990), 55-61.

[35] S. Peng, Stochastic Hamilton Jacobi Bellman equations, SIAM Journal on Control and Optimization, 30 (1992), 284-304. 
[36] S. Peng, A generalized dynamic programming principle and HamiltonJacobi-Bellman equation, Stochastics and Stochastic Reports, 38 (1992), 119-134.

[37] S. Peng, Backward stochastic differential equations and exact controllability of stochastic control systems, Progress in natural science, 4 (1994), 274-284.

[38] D. Revuz and M. Yor, Continuous martingales and Brownian motion, Third Edition (1999), Springer.

[39] Y. Wang and X. Wang, Adapted solutions of backward SDE with nonlipschitz coefficients, Chinese J. Appl. Probab. Statist., 19 (2003), 245251 (in chinese).

[40] Y. Wang and Z. Huang, Backward stochastic differrential equations with non-Lipschitz coefficients, Statistics \& Probability Letters, 79 (2009) 1438-1443.

[41] Y. Wang, BSDEs with general filtration driven by Lévy processes, and an application in stochastic controllability, Systems $\& 5$ Control Letters, 62 (2013), 242-247.

[42] J. Yong and and X. Y. Zhou, 1999, Stochastic Controls: Hamiltonian Systems and HJB Equations, Springer, 1999.

[43] J. Yong, Completeness of security markets and solvability of linear backward stochastic differential equations, J. Math. Anal. Appl., 319 (2006), 333-356.

[44] J. Yong, Remarks on some short rate term structure models, Journal of Industrial and Management Optimization, 2 (2006), 119-134.

[45] S. Zheng and S. Li, Representation theorems for generators of BSDEs with monotonic and convex growth generators, Statistics \& Probability Letters, 97 (2015), 197-205.

[46] C. Zhigang and Y. Jia-An, A comparison theorem for solutions of backward stochastic differential equations, Advances in Mathematics, 28 (1999), 304-308. 